\title{
Internally Calabi-Yau algebras and cluster-tilting objects
}

\author{
Matthew Pressland ${ }^{1}$ iD
}

Received: 4 August 2016 / Accepted: 1 December 2016 / Published online: 3 January 2017

(C) The Author(s) 2017. This article is published with open access at Springerlink.com

\begin{abstract}
We describe what it means for an algebra to be internally $d$-Calabi-Yau with respect to an idempotent. This definition abstracts properties of endomorphism algebras of $(d-1)$-cluster-tilting objects in certain stably $(d-1)$-Calabi-Yau Frobenius categories, as observed by Keller-Reiten. We show that an internally $d$-Calabi-Yau algebra satisfying mild additional assumptions can be realised as the endomorphism algebra of a $(d-1)$-clustertilting object in a Frobenius category. Moreover, if the algebra satisfies a stronger 'bimodule' internally $d$-Calabi-Yau condition, this Frobenius category is stably $(d-1)$-Calabi-Yau. We pay special attention to frozen Jacobian algebras; in particular, we define a candidate bimodule resolution for such an algebra, and show that if this complex is indeed a resolution, then the frozen Jacobian algebra is bimodule internally 3-Calabi-Yau with respect to its frozen idempotent. These results suggest a new method for constructing Frobenius categories modelling cluster algebras with frozen variables, by first constructing a suitable candidate for the endomorphism algebra of a cluster-tilting object in such a category, analogous to Amiot's construction in the coefficient-free case.
\end{abstract}

Keywords Calabi-Yau algebra · Cluster algebra · Cluster-tilting object · Frobenius category $\cdot$ Jacobian algebra $\cdot$ Quiver with potential

Mathematics Subject Classification $13 \mathrm{~F} 60 \cdot 16 \mathrm{G} 20 \cdot 16 \mathrm{G} 50 \cdot 18 \mathrm{E} 30$

\section{Introduction}

Cluster categories, first introduced in special cases by Buan-Marsh-Reineke-ReitenTodorov [10] and later generalised by Amiot [1], are certain Hom-finite 2-Calabi-Yau triangulated categories that model the combinatorics of cluster algebras without frozen vari-

Matthew Pressland

mdpressland@bath.edu

http://guests.mpim-bonn.mpg.de/mdp33/

1 Max-Planck-Institut für Mathematik, Vivatsgasse 7, 53111 Bonn, Germany 
ables. In particular, a cluster category $\mathcal{C}$ contains cluster-tilting objects, which are objects $T$ satisfying

$$
\text { add } T=\left\{X \in \mathcal{C}: \operatorname{Ext}_{\mathcal{C}}^{1}(T, X)=0\right\}=\left\{X \in \mathcal{C}: \operatorname{Ext}_{\mathcal{C}}^{1}(X, T)=0\right\} .
$$

Basic cluster-tilting objects, whose summands in any direct sum decomposition are pairwise non-isomorphic, model the clusters of the cluster algebra; from now on, any time we refer to a cluster-tilting object, we assume it to be basic.

The main reason that the cluster-tilting objects of $\mathcal{C}$ can be said to model the clusters of a cluster algebra is that, as with a cluster, it is possible to pass from one cluster-tilting object to another by a process of mutation. For any indecomposable summand $T_{i}$ of a cluster-tilting object $T$, there exists a unique indecomposable $T_{i}^{\prime} \in \mathcal{C}$, not isomorphic to $T_{i}$, such that $T / T_{i} \oplus T_{i}^{\prime}$ is a cluster-tilting object. Moreover, $T_{i}^{\prime}$ can be computed by either of the exchange triangles

$$
\begin{aligned}
& T_{i} \stackrel{f}{\longrightarrow} X_{i} \longrightarrow T_{i}^{\prime} \longrightarrow T_{i}[1], \\
& T_{i}^{\prime} \longrightarrow Y_{i} \stackrel{g}{\longrightarrow} T_{i} \longrightarrow T_{i}^{\prime}[1],
\end{aligned}
$$

in which $f$ is a minimal left $\operatorname{add}\left(T / T_{i}\right)$-approximation of $T_{i}$, and $g$ is a minimal right $\operatorname{add}\left(T / T_{i}\right)$-approximation of $T_{i}$. Choosing an initial cluster-tilting object $T^{0}=\bigoplus_{i=1}^{n} T_{i}^{0}$ of $\mathcal{C}$ yields a cluster character $[13,36] \varphi: \mathcal{C} \rightarrow \mathscr{A}(Q, \mathbf{x})$ from the objects of $\mathcal{C}$ to the cluster algebra $\mathscr{A}(Q, \mathbf{x})$ with initial seed given by the quiver $Q$ of $\operatorname{End}_{\mathcal{C}}(T)^{\text {op }}$ with cluster variables $x_{i}=\varphi_{T_{i}^{0}}$. The exchange triangles correspond to the exchange relations

$$
\varphi_{T_{i}} \varphi_{T_{i}^{\prime}}=\varphi_{X_{i}}+\varphi_{Y_{i}}
$$

in this cluster algebra. The cluster variables of $\mathscr{A}(Q, \mathbf{x})$ are precisely the elements of the form $\varphi_{M}$ for $M$ an indecomposable reachable rigid object of $\mathcal{C}$, where rigid means that $\operatorname{Ext}_{\mathcal{C}}^{1}(M, M)=0$, and reachable means that $M$ is a summand of a reachable cluster-tilting object, i.e. one obtained from $T^{0}$ by a finite sequence of mutations. The clusters are the sets of the form $\left\{\varphi_{T_{1}}, \ldots, \varphi_{T_{n}}\right\}$ for $T=\bigoplus_{i=1}^{n} T_{i}$ reachable cluster-tilting in $\mathcal{C}$.

For a more thorough introduction to the theory of cluster algebras and their categorification, we recommend Keller's survey [32].

The categorification of cluster algebras by cluster categories has proved to be very useful in studying their combinatorics, since the cluster category $\mathcal{C}$ can be considered more globally than the associated cluster algebra $\mathscr{A}(Q, \mathbf{x})$. For example, to identify clusters or cluster variables of $\mathscr{A}(Q, \mathbf{x})$, one usually has to find a sequence of mutations from a known cluster, which is a highly computationally intensive procedure. By contrast, cluster-tilting or rigid objects of $\mathcal{C}$ are characterised intrinsically.

For this reason, it would be extremely useful to be able to more readily categorify cluster algebras that do have frozen variables, particularly as most of the examples occurring in nature, such as those on the coordinate rings of partial flag varieties and their unipotent cells, as studied by Geiß-Leclerc-Schröer [19], are of this type. The natural candidate for such a categorification is a stably 2-Calabi-Yau Frobenius category, as we now describe.

A Frobenius category is an exact category with enough projective and injective objects, such that these two classes of objects coincide. If $\mathcal{E}$ is a Frobenius category, then the stable category $\underline{\mathcal{E}}=\mathcal{E} / \operatorname{proj} \mathcal{E}$ is triangulated by a famous result of Happel [23, §I.2]. It is immediate from the definition that if $T \in \mathcal{E}$ is cluster-tilting, then $\operatorname{proj} \mathcal{E} \subset$ add $T$. In this case, we must have proj $\mathcal{E}=$ add $P$ for some object $P=\bigoplus_{i=r+1}^{n} T_{i}$; the intention is that the objects $T_{i}$ for $r<i \leq n$, which occur as summands of every cluster-tilting object of $\mathcal{E}$, will correspond to the 
frozen variables of a cluster algebra. Factoring out proj $\mathcal{E}$ corresponds to setting these frozen variables to 1 in the cluster algebra, to recover a cluster algebra without frozen variables. Thus the stable category $\underline{\mathcal{E}}$ should be a cluster category-in particular it should be 2-Calabi-Yau.

Such categorifications have been described for certain cluster algebras relating to partial flag varieties, for example by Geiss-Leclerc-Schröer [19], Jensen-King-Su [28] and Demonet-Iyama [14], and we will recall some of these constructions in Sect. 3. Nájera Chávez [35] has also categorified finite type cluster algebras with 'universal' coefficients. However, in all cases, the construction of the category depends on having at least a partial understanding of the overall structure of the cluster algebra. Thus the methods of these papers cannot be easily abstracted to produce categorifications for more general cluster algebras with frozen variables.

The main aim of this paper is to consider how one might be able to produce a categorification of a cluster algebra with frozen variables without understanding this global structure, instead starting only from the data of a single seed, which is how a cluster algebra is usually specified. This is analogous to Amiot's construction of cluster categories in the case that there are no frozen variables; given a seed, one has to find a rigid potential on the quiver of the seed such that the resulting Jacobian algebra (see Sect. 5) is finite dimensional, and then Amiot provides a general recipe for constructing a categorification of the cluster algebra. Our construction in the case that there are frozen variables is similar, but requires more data satisfying more conditions. Given the seed of a cluster algebra with frozen variables, we take its quiver, and aim to add arrows between frozen variables and choose a potential such that the resulting frozen Jacobian algebra satisfies a number of conditions, most importantly that of being bimodule internally 3-Calabi-Yau. If this can be achieved, the general machinery developed in this paper can take over to produce the desired Frobenius category, from which the frozen Jacobian algebra can be recovered as the endomorphism algebra of a cluster-tilting object.

For most of the paper, we will in fact work in a higher level of generality, and construct stably $d$-Calabi-Yau Frobenius categories admitting $d$-cluster-tilting objects (Definition 3.2); setting $d=2$ recovers the definition of cluster-tilting given above.

The structure of the paper is as follows. In Sect. 2 we say what it means for an algebra to be internally $d$-Calabi-Yau with respect to an idempotent $e$ (Definition 2.1), and also make a stronger, more symmetric, definition of bimodule internally $d$-Calabi-Yau with respect to $e$ (Definition 2.4). An algebra $A$ satisfying either of these definitions has, in particular, finite global dimension, and a duality

$$
\mathrm{DExt}_{A}^{i}(M, N)=\operatorname{Ext}_{A}^{d-i}(N, M)
$$

for any pair $M$ and $N$ of $A$-modules such that $M$ is finite dimensional, $e M=0$ and both $M$ and $N$ are perfect when considered as stalk complexes in the bounded derived category of $A$. In much of the paper we will also assume that $A$ is Noetherian, in which case this last condition reduces to $M$ and $N$ being finitely generated.

In Sect. 3, we introduce the class of Frobenius $m$-cluster categories for $m \geq 1$, and exploit a result of Keller-Reiten [33] to show that the endomorphism algebra of an $m$ cluster-tilting object in a Frobenius $m$-cluster category is internally $(m+1)$-Calabi-Yau. A Morita-type theorem of Iyama-Kalck-Wemyss-Yang [29] implies that any Frobenius $m$ cluster category admitting an $m$-cluster-tilting object with Noetherian endomorphism algebra is equivalent to the category of Gorenstein projective modules over some Iwanaga-Gorenstein ring (Definition 3.8), and so we pay special attention to categories of this form. We also give brief descriptions of some important families of Frobenius $m$-cluster categories already appearing in the literature. 
In Sect. 4, we show that an algebra $A$ that is internally $d$-Calabi-Yau with respect to an idempotent $e$ (on both sides) for some $d \geq 2$, and satisfies mild additional assumptions, necessarily arises as the endomorphism algebra of a $(d-1)$-cluster-tilting object in some Frobenius category determined by $A$ and $e$. Precisely, we prove the following theorem.

Theorem 1 (Theorem 4.1) Let A be a Noetherian algebra, and let e $\in$ A be an idempotent such that $A /\langle e\rangle$ is finite dimensional and both $A$ and $A^{\mathrm{op}}$ are internally $d$-Calabi-Yau with respect to $e$. Write $B=e$ Ae and $\underline{A}=A /\langle e\rangle$. Then

(i) B is Iwanaga-Gorenstein with Gorenstein dimension at most d, so

$$
\operatorname{GP}(B)=\left\{X \in \bmod B: \operatorname{Ext}_{B}^{i}(X, B)=0, i>0\right\}
$$

is a Frobenius category,

(ii) $e A$ is $(d-1)$-cluster-tilting in $\mathrm{GP}(B)$, and

(iii) there are natural isomorphisms $\operatorname{End}_{B}(e A)^{\mathrm{op}} \stackrel{\sim}{\rightarrow} A$ and $\operatorname{End}_{\underline{\mathrm{GP}}(B)}(e A)^{\mathrm{op}} \stackrel{\sim}{\rightarrow} \underline{A}$.

Under the stronger assumption that $A$ is bimodule internally $d$-Calabi-Yau with respect to $e$, we can show more.

Theorem 2 (Theorem 4.10) Let A be a Noetherian algebra and let $e \in A$ be an idempotent such that $A /\langle e\rangle$ is finite dimensional, and $A$ is bimodule internally $d$-Calabi-Yau with respect to $e$. Write $B=e$ Ae. Then all of the conclusions of Theorem 1 hold, and moreover $\mathrm{GP}(B)$ is $(d-1)$-Calabi-Yau.

While, in general, checking that an algebra is internally $d$-Calabi-Yau with respect to an idempotent can be very difficult, there is more hope in the case that $A$ is a frozen Jacobian algebra (Definition 5.1). Such an algebra is presented via a quiver with relations, in which the relations are dual to some of the arrows; the arrows which do not have any corresponding relations are called frozen, and their end-points are frozen vertices. In Sect. 5, we show that the required Calabi-Yau symmetry can be deduced from the exactness of a combinatorially defined complex $\mathbf{P}(A) \rightarrow A$ (Definition 5.4), generalising work of Ginzburg [20] for Jacobian algebras. More precisely, we prove the following.

Theorem 3 (Theorem 5.6) If $A$ is a frozen Jacobian algebra such that $\mathbf{P}(A)$ is quasiisomorphic to $A$, then $A$ is bimodule internally 3-Calabi-Yau with respect to the idempotent $e=\sum_{v \in F_{0}} e_{v}$, where $F_{0}$ denotes the set of frozen vertices.

The results of Sect. 5 are inspired by work of Broomhead [7] on consistent dimer models (also known as brane tilings or bipartite field theories) on closed surfaces, which has applications to theoretical physics. We expect our results to have consequences for the more recent theory of dimer models on surfaces with boundary, studied for example by Franco [17], and which has already appeared in the context of cluster categorification for the Grassmannian in work of Baur-King-Marsh [5]. Dimer models on surfaces with boundary have also appeared, under the name 'plabic graphs', in work of Postnikov [37] on the positive Grassmannian, and in recent work of Goncharov [21].

Throughout, we fix a field $\mathbb{K}$, and assume all categories are $\mathbb{K}$-linear and all algebras are associative $\mathbb{K}$-algebras with unit. If $V$ is a $\mathbb{K}$-vector space, we write $\mathrm{D} V=\operatorname{Hom}_{\mathbb{K}}(V, \mathbb{K})$ for the dual space. All modules are unital, and are left modules unless otherwise indicated. Given an algebra $A$, we denote by $\operatorname{Mod} A$ the category of all $A$-modules, and by $\bmod A$ the category of finitely generated $A$-modules. Given an object $X$ of an exact category $\mathcal{E}$, we denote by add $X$ the full subcategory of $\mathcal{E}$ with objects isomorphic to finite direct sums 
of direct summands of $X$. We say an algebra $A$ is Noetherian if it is Noetherian as both a left and right module over itself; this is stronger than requiring it to be Noetherian as an $A$-bimodule. We denote by $\mathcal{D} A$ and $\mathcal{D}^{b} A$ the derived and bounded derived categories of $A$, and by $\mathcal{D}_{\mathrm{fd}}(A)$ the full subcategory of $\mathcal{D}^{b} A$ consisting of objects with finite dimensional total cohomology. We denote by per $A$ the thick triangulated subcategory of $\mathcal{D}^{b} A$ generated by $A$ (or equivalently by the finitely generated projective $A$-modules). Objects of per $A$ are called perfect, and we will say that an $A$-module $M$ is perfect if the stalk complex with $M$ in degree 0 is perfect.

\section{Internally $d$-Calabi-Yau algebras}

This section introduces our main definitions.

Definition 2.1 Let $A$ be a $\mathbb{K}$-algebra, $e$ an idempotent of $A$, and $d$ a non-negative integer. We say $A$ is internally $d$-Calabi-Yau with respect to $e$ if

(i) gl. $\operatorname{dim} A \leq d$, and

(ii) for each $i \in \mathbb{Z}$, there is a functorial duality

$$
\operatorname{DExt}_{A}^{i}(M, N)=\operatorname{Ext}_{A}^{d-i}(N, M)
$$

where $M$ and $N$ are perfect $A$-modules such that $M$ is also a finite dimensional $A /\langle e\rangle$ module, where $\langle e\rangle=A e A$ is the two-sided ideal of $A$ generated by $e$.

Recall that a module $M$ is perfect if the stalk complex with $M$ in degree 0 is perfect, i.e. quasi-isomorphic to a bounded complex of finitely generated projective $A$-modules. Thus a perfect module is always both finitely generated and finitely presented, but the converse statements need not hold. If $A$ is Noetherian with finite global dimension (which will be the case for much of the paper, including the main theorems in Sect. 4), then an A-module is perfect if and only it is finitely generated. We will also consider other situations in which perfectness is equivalent to a more familiar notion, and will try to indicate these as they arise.

Note the lack of symmetry in the finiteness conditions on $M$ and $N$; cf. [31, Lem. 4.1]. These conditions are imposed in order to force the space $\operatorname{Ext}_{A}^{d-i}(N, M)$ on the right-hand side of the duality formula to be finite dimensional over $\mathbb{K}$.

Remark 2.2 If $A$ is internally $d$-Calabi-Yau with respect to $e$ then it is internally $d$-CalabiYau with respect to $e+e^{\prime}$ for any idempotent $e^{\prime} \in A$ orthogonal to $e$. An algebra $A$ is $d$-Calabi-Yau if and only if it is internally $d$-Calabi-Yau with respect to 0 , and hence with respect to every idempotent. At the other extreme, $A$ is internally $d$-Calabi-Yau with respect to 1 if and only if gl. $\operatorname{dim} A \leq d$.

Remark 2.3 A finite dimensional algebra $A$ is internally $d$-Calabi-Yau with respect to $e$ if and only if the same is true of $A^{\mathrm{op}}$; since $A$ is finite dimensional, it is Noetherian, so gl. $\operatorname{dim} A=$ gl. $\operatorname{dim} A^{\text {op }}$ [40, Ex. 4.1.1], and $\mathrm{D}=\operatorname{Hom}_{\mathbb{K}}(-, \mathbb{K})$ induces an equivalence $\bmod A^{\mathrm{op}} \stackrel{\sim}{\rightarrow}(\bmod A)^{\mathrm{op}}$ yielding the required functorial duality for $A^{\mathrm{op}}$.

Definition 2.1 is not necessarily left-right symmetric in this way if $A$ is infinite dimensional, so we will also make a stronger definition that does have this property. Denote by $A^{\varepsilon}=A \otimes_{\mathbb{K}}$ $A^{\text {op }}$ the enveloping algebra of $A$, so that an $A$-bimodule is the same as an $A^{\varepsilon}$-module. Write $\Omega_{A}=\mathbf{R H o m}_{A^{\varepsilon}}\left(A, A^{\varepsilon}\right)$. We view $\Omega_{A}$ as a complex in $\mathcal{D} A^{\varepsilon}$ via the 'inner' multiplication on 
$A^{\varepsilon}$; for any homomorphism $f: M \rightarrow A^{\varepsilon}$ of $A$-bimodules such that $f(m)=u \otimes v$ and any $x \otimes y \in A^{\varepsilon}$, let $x f y(m)=u y \otimes x v$.

Recall [2, Defn. 2.1] that $A$ is said to be bimodule $d$-Calabi-Yau if $A \in \operatorname{per} A^{\varepsilon}$ (i.e. $A$ is quasi-isomorphic to a bounded complex of finitely generated projective $A$-bimodules) and there is an isomorphism $A \stackrel{\sim}{\rightarrow} \Omega_{A}[d]$ in $\mathcal{D} A^{\varepsilon}$. This definition is slightly weaker than that of Ginzburg [20, 3.2.5], as we will not need to impose any 'self-duality' condition on the isomorphism.

If $A$ is an algebra with quotient $\underline{A}$, write $\mathcal{D}_{\underline{A}}(A)$ for the full subcategory of $\mathcal{D} A$ consisting of complexes with homology groups in $\operatorname{Mod} \underline{A}$, and $\mathcal{D}_{\mathrm{fd}, \underline{A}}(A)$ for the full subcategory of $\mathcal{D}_{\underline{A}}(A)$ consisting of objects with finite dimensional total cohomology.

Definition 2.4 An algebra $A$ is bimodule internally $d$-Calabi-Yau with respect to an idempotent $e \in A$ if

(i) p. $\operatorname{dim}_{A^{\varepsilon}} A \leq d$,

(ii) $A \in \operatorname{per} A^{\varepsilon}$, and

(iii) there exists a triangle

$$
A \stackrel{\psi}{\longrightarrow} \Omega_{A}[d] \longrightarrow C \longrightarrow A[1]
$$

in $\mathcal{D} A^{\varepsilon}$, such that

$$
\mathbf{R H o m}_{A}(C, M)=0=\mathbf{R H o m}_{A^{\text {op }}}(C, N)
$$

for any $M \in \mathcal{D}_{\mathrm{fd}, \underline{A}}(A)$ and $N \in \mathcal{D}_{\mathrm{fd}, \underline{A}^{\mathrm{op}}}\left(A^{\mathrm{op}}\right)$, where $\underline{A}=A /\langle e\rangle$.

Remark 2.5 An algebra $A$ is bimodule internally $d$-Calabi-Yau with respect to 0 if and only if $\psi$ can be chosen to be a quasi-isomorphism, or equivalently if $A$ is bimodule $d$-Calabi-Yau. In this case, (i) follows from (ii) and (iii) [2, Prop. 2.5(b)]. When $e \neq 0$, this implication does not hold, and so we must make the stronger condition part of the definition. Note also that (i) does not imply (ii), since it asserts only that $A$ has a finite resolution by projective $A^{\varepsilon}$-modules, whereas (ii) requires additionally that these modules are finitely generated.

An algebra $A$ is bimodule internally $d$-Calabi-Yau with respect to 1 if and only if p. $\operatorname{dim}_{A^{\varepsilon}} A \leq d$ and $A \in \operatorname{per} A^{\varepsilon}$; in this case $\underline{A}=0$, so condition (iii) is satisfied for any $\psi$.

Remark 2.6 There is an isomorphism $A^{\varepsilon} \stackrel{\sim}{\rightarrow}\left(A^{\text {op }}\right)^{\varepsilon}$ given by reversing the order of the tensor product. The resulting equivalence $\bmod A^{\varepsilon} \stackrel{\sim}{\rightarrow} \bmod \left(A^{\mathrm{op}}\right)^{\varepsilon}$ takes $A$ to $A^{\text {op }}$ (and $\underline{A}$ to $\underline{A}^{\mathrm{op}}$ ). As a result, Definition 2.4 is left-right symmetric, meaning that $A$ is bimodule internally $d$-Calabi-Yau with respect to $e$ if and only if the same is true of $A^{\mathrm{op}}$.

The following lemma, due to Keller, allows us to recover dualities of extension groups between $A$-modules from bimodule properties of $A$.

Lemma 2.7 ([31, Lem. 4.1]) Assume $A \in$ per $A^{\varepsilon}$. For all objects $M, N \in \mathcal{D} A$ such that $M$ has finite dimensional total cohomology, there is a functorial isomorphism

$$
\operatorname{DHom}_{\mathcal{D} A}(M, N) \stackrel{\sim}{\rightarrow} \operatorname{Hom}_{\mathcal{D} A}\left(\Omega_{A} \stackrel{\mathbf{L}}{\otimes}_{A} N, M\right) .
$$

If $A$ is bimodule $d$-Calabi-Yau, then $\Omega_{A} \cong A[-d]$ in $\mathcal{D} A^{\varepsilon}$. It then follows from Lemma 2.7 that for any $M, N \in \operatorname{Mod} A$, with $M$ finite dimensional, we have

$$
\operatorname{DExt}_{A}^{i}(M, N)=\operatorname{DHom}_{\mathcal{D} A}(M, N[i])
$$




$$
\begin{aligned}
& \cong \operatorname{Hom}_{\mathcal{D} A}\left(\Omega_{A} \otimes_{A}^{\mathbf{L}} N[i], M\right) \\
& \cong \operatorname{Hom}_{\mathcal{D} A}(N[i-d], M) \\
& =\operatorname{Ext}_{A}^{d-i}(N, M) .
\end{aligned}
$$

We now use Lemma 2.7 to prove a similar result for bimodule internally $d$-Calabi-Yau algebras.

Theorem 2.8 Let $A$ be bimodule internally $d$-Calabi-Yau with respect to $e$, and let $\underline{A}=$ $A /\langle e\rangle$. Then for any $N \in \mathcal{D} A$ and any $M \in \mathcal{D}_{\mathrm{fd}, \underline{A}}(A)$, we have a functorial isomorphism

$$
\operatorname{DHom}_{\mathcal{D} A}(M, N)=\operatorname{Hom}_{\mathcal{D} A}(N[-d], M) .
$$

Proof Pick a triangle

$$
A[-d] \stackrel{\psi}{\longrightarrow} \Omega_{A} \longrightarrow C \longrightarrow A[1-d]
$$

by applying $[-d]$ to a triangle as in Definition 2.4. Applying $-\stackrel{\mathbf{L}}{\otimes}{ }_{A} N$ yields a triangle

$$
N[-d] \longrightarrow \Omega_{A} \stackrel{\mathbf{L}}{\otimes}{ }_{A} N \longrightarrow C \stackrel{\mathbf{L}}{\otimes}_{A} N \longrightarrow N[1-d]
$$

in $\mathcal{D} A$. Now apply $\mathbf{R H o m}_{A}(-, M)$, to get a triangle

$$
\mathbf{R H o m}_{A}\left(\Omega_{A} \otimes_{A}^{\mathbf{L}} N, M\right) \longrightarrow \mathbf{R H o m}_{A}(N[-d], M)
$$

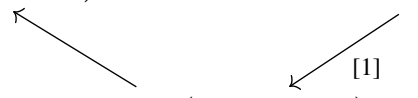

Since $M \in \mathcal{D}_{\mathrm{fd}, \underline{A}}(A)$, we have $\operatorname{RHom}_{A}(C, M)=0$ by definition, and so

$$
\mathbf{R H o m}_{A}\left(C \stackrel{\mathbf{L}}{\otimes}{ }_{A} N, M\right)=\mathbf{R H o m}_{A}\left(N, \mathbf{R H o m}_{A}(C, M)\right)=0 .
$$

Thus $\mathbf{R H o m}_{A}\left(\Omega_{A} \stackrel{\mathbf{L}}{\otimes}{ }_{A} N, M\right) \cong \mathbf{R H o m}_{A}(N[-d], M)$. We obtain the desired result by taking 0-th cohomology and applying Lemma 2.7 .

Corollary 2.9 If A is bimodule internally d-Calabi-Yau with respect to e, then it is internally $d$-Calabi-Yau with respect to e, and moreover we have a functorial isomorphism

$$
\mathrm{DExt}_{A}^{i}(M, N)=\operatorname{Ext}_{A}^{d-i}(N, M)
$$

for any finite dimensional $M \in \bmod A /\langle e\rangle$ and any $N \in \operatorname{Mod} A$, which need not be perfect as A-modules.

Proof Since p. $\operatorname{dim}_{A^{\varepsilon}} A \leq d$, there is an exact sequence

$$
0 \longrightarrow P_{d} \longrightarrow \cdots \longrightarrow P_{1} \longrightarrow P_{0} \longrightarrow A \longrightarrow 0
$$


of $A$-bimodules, in which each $P_{i}$ is a projective $A$-bimodule. If $X$ is any $A$-module, then $P_{i} \otimes_{A} X$ is a projective $A$-module, and so applying $-\otimes_{A} X$ to the above sequence gives a projective resolution

$$
0 \longrightarrow P_{d} \otimes_{A} X \longrightarrow \cdots \longrightarrow P_{1} \otimes_{A} X \longrightarrow P_{0} \otimes_{A} X \longrightarrow X \longrightarrow 0
$$

of $X$. It follows that gl. $\operatorname{dim} A \leq d$.

Now by Theorem 2.8, if $N \in \operatorname{Mod} A$ and $M \in \bmod A /\langle e\rangle$ is finite dimensional, we have

$$
\begin{aligned}
\operatorname{DExt}_{A}^{i}(M, N) & =\operatorname{DHom}_{\mathcal{D} A}(M, N[i]) \\
& =\operatorname{Hom}_{\mathcal{D} A}(N[i-d], M) \\
& =\operatorname{Ext}_{A}^{d-i}(N, M) .
\end{aligned}
$$

In particular, this duality applies when $M$ and $N$ are perfect $A$-modules, so $A$ is internally $d$-Calabi-Yau with respect to $e$.

While Theorem 2.8 and Corollary 2.9 show that bimodule internally Calabi-Yau algebras have stronger properties than those required by the definition of internally Calabi-Yau, with the duality formula applying to a wider class of objects, we do not currently know of any examples of internally Calabi-Yau algebras which are not bimodule internally Calabi-Yau. It seems unlikely that the two classes of algebras coincide, but it is tempting to speculate based on work of Bocklandt [6] that if $A$ is an internally Calabi-Yau algebra admitting a suitable grading, then it is also bimodule internally Calabi-Yau.

Example 2.10 Consider the algebra $A=\mathbb{K} Q / I$ given by the quiver

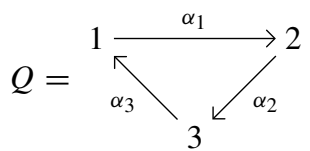

with ideal of relations $I=\left\langle\alpha_{2} \alpha_{1}, \alpha_{1} \alpha_{3}\right\rangle$. This is an example of a frozen Jacobian algebra; see Definition 5.1 and Example 5.3. One can check (for example, by Theorem 5.6 below) that $A$ is bimodule internally 3-Calabi-Yau with respect to $e_{1}+e_{2}$, and so both $A$ and $A^{\text {op }}$ are internally 3-Calabi-Yau with respect to this idempotent.

Similarly, the algebra $A^{\prime}=\mathbb{K} Q^{\prime} / I^{\prime}$ given by the quiver

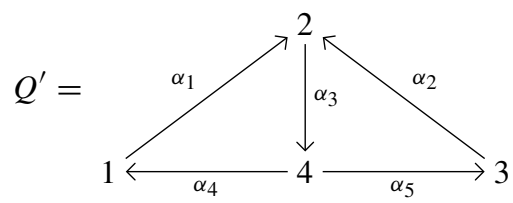

with ideal of relations $I^{\prime}=\left\langle\alpha_{3} \alpha_{1}, \alpha_{1} \alpha_{4}-\alpha_{2} \alpha_{5}, \alpha_{3} \alpha_{2}\right\rangle$ is bimodule internally 3-Calabi-Yau with respect to $e_{1}+e_{2}+e_{3}$.

\section{Frobenius $m$-cluster categories}

This section is devoted to describing a class of categories, which we term Frobenius $m$-cluster categories, providing us with a rich source of internally $(m+1)$-Calabi-Yau algebras. Indeed, certain categories in this class motivated Definition 2.1. 
Recall that an exact category $\mathcal{E}$ is Frobenius if it has enough projective objects and enough injective objects, and $\operatorname{proj} \mathcal{E}=\operatorname{inj} \mathcal{E}$. By a famous result of Happel [23, §I.2], the stable category $\underline{\mathcal{E}}=\mathcal{E} / \operatorname{proj} \mathcal{E}$ is triangulated, with suspension given by the inverse syzygy $\Omega^{-1}$, taking an object to the cokernel of an injective hull.

Definition 3.1 Let $\mathcal{E}$ be a Frobenius category, and let $m \geq 1$. We say that $\mathcal{E}$ is stably $m$ Calabi-Yau if its stable category $\underline{\mathcal{E}}$ is $m$-Calabi-Yau, meaning that $\underline{\mathcal{E}}$ is Hom-finite, and there is a functorial duality

$$
\mathrm{D} \underline{\operatorname{Hom}}_{\mathcal{E}}(X, Y)=\underline{\operatorname{Hom}}_{\mathcal{E}}\left(Y, \Omega^{-m} X\right)
$$

for all $X, Y \in \mathcal{E}$. Here $\underline{\operatorname{Hom}}_{\mathcal{E}}(X, Y)$ denotes the space of morphisms $X$ to $Y$ in $\underline{\mathcal{E}}$.

Definition 3.2 Let $\mathcal{E}$ be an exact category, and let $\mathcal{T} \subset \mathcal{E}$ be a full and functorially finite subcategory closed under direct sums and direct summands. We say $\mathcal{T}$ is an $m$-cluster-tilting subcategory if

$$
\left\{X \in \mathcal{E}: \operatorname{Ext}_{\mathcal{E}}^{i}(X, \mathcal{T})=0,0<i<m\right\}=\mathcal{T}=\left\{X \in \mathcal{E}: \operatorname{Ext}_{\mathcal{E}}^{i}(\mathcal{T}, X)=0,0<i<m\right\} .
$$

Here ' $\operatorname{Ext}_{\mathcal{E}}^{i}(X, \mathcal{T})=0$ ' is taken to mean ' $\operatorname{Ext}_{\mathcal{E}}^{i}(X, T)=0$ for all $T \in \mathcal{T}$ '. We say an object $T \in \mathcal{E}$ is an $m$-cluster-tilting object if add $T$ is an $m$-cluster-tilting subcategory.

If $\mathcal{E}$ is a Frobenius category, then for any $X, Y \in \mathcal{E}$ and $i>0$, we have

$$
\operatorname{Ext}_{\mathcal{E}}^{i}(X, Y)=\underline{\operatorname{Hom}}_{\mathcal{E}}\left(X, \Omega^{-i} Y\right) .
$$

Thus if $\mathcal{E}$ is stably $m$-Calabi-Yau, the two equalities appearing in Definition 3.2 are equivalent to one another.

Definition 3.3 Let $\mathcal{E}$ be a Frobenius category, and $m \geq 1$. Then $\mathcal{E}$ is called a Frobenius $m$ cluster category if it is idempotent complete, stably $m$-Calabi-Yau, and gl. $\operatorname{dim} \operatorname{End}_{\mathcal{E}}(T)^{\mathrm{op}} \leq$ $m+1$ for any $m$-cluster-tilting object $T$, of which there is at least one. If these properties hold for $m=2$, then $\mathcal{E}$ will be called simply a Frobenius cluster category.

Note that while $\underline{\mathcal{E}}$ is Hom-finite for any Frobenius $m$-cluster category $\mathcal{E}$, we do not assume that $\mathcal{E}$ itself is Hom-finite (cf. Example 3.12). By the following result, which follows immediately from a theorem of Keller-Reiten [33, 5.4], Frobenius $m$-cluster categories provide a rich source of examples of internally Calabi-Yau algebras.

Theorem 3.4 Let $\mathcal{E}$ be a Frobenius m-cluster category, let $T \in \mathcal{E}$ be a basic m-clustertilting object, and write $A=\operatorname{End}_{\mathcal{E}}(T)^{\mathrm{op}}$. Let $P$ be a maximal projective-injective summand of $T$, and let $e \in A$ be the idempotent given by projection onto $P$. Then $A$ is internally $(m+1)$-Calabi-Yau with respect to $e$.

Proof Let $M$ and $N$ be perfect $A$-modules, and assume that $M$ is a finite dimensional $A /\langle e\rangle$ module. Then by $[33,5.4]$, there is a functorial duality

$$
\begin{aligned}
\operatorname{Ext}_{A}^{i}(M, N) & =\operatorname{Hom}_{\text {per } A}(M[-i], N) \\
& =\operatorname{DHom}_{\text {per } A}(N, M[m+1-i])=\operatorname{DExt}_{A}^{m+1-i}(N, M)
\end{aligned}
$$

for all $i$. Since $\mathrm{gl} \lim A \leq m+1$ by assumption, we have the desired result. 
Remark 3.5 If $\mathcal{E}$ is a Frobenius $m$-cluster category such that $A=\operatorname{End}_{\mathcal{E}}(T)^{\mathrm{op}}$ is Noetherian for any $m$-cluster tilting object $T$, then $\mathcal{E}^{\text {op }}$ is a Frobenius $m$-cluster category with the same $m$-cluster-tilting objects as $\mathcal{E}$; the extra assumption is used to ensure that

$$
\text { gl. } \operatorname{dim} A^{\text {op }}=\text { gl. } \operatorname{dim} A \leq m+1 .
$$

Thus, under these circumstances, $A^{\mathrm{op}}$ is also internally $(m+1)$-Calabi-Yau with respect to $e$

In the context of Theorem 3.4, we have a more straightforward characterisation of the $A /\langle e\rangle$-modules that are perfect as $A$-modules, again coming from Keller-Reiten's work.

Proposition 3.6 Let $\mathcal{E}$ be a Frobenius $m$-cluster category, let $T \in \mathcal{E}$ be a basic $m$-clustertilting object, and write $A=\operatorname{End}_{\mathcal{E}}(T)^{\mathrm{op}}$. Let $P$ be a maximal projective-injective summand of $T$, let $e \in A$ be the idempotent given by projection onto $P$ and write $\underline{A}=A /\langle e\rangle$. Then an A-module $M$ is perfect as an A-module if and only if it is finitely generated.

Proof Let $M \in \bmod \underline{A}$. If $M$ is perfect over $A$, then it is in particular finitely generated over $A$, and thus (by the same generating set) over $\underline{A}$. Since $\underline{A}=\underline{\operatorname{End}}_{\mathcal{E}}(T)^{\text {op }}$ and $\underline{\mathcal{E}}$ is Hom-finite, $\underline{A}$ is finite dimensional. It follows that any finitely generated $\underline{A}$-module is finitely presented over $\underline{A}$, and thus perfect over $A$ by $[33,5.4(\mathrm{c})]$.

The following proposition, which is based on work of Iyama [25, §2], [26, Thm. 3.6.2], gives sufficient conditions on a Frobenius category $\mathcal{E}$ for us to conclude that $\operatorname{gl}$. $\operatorname{dim} \operatorname{End}_{\mathcal{E}}(T)^{\mathrm{op}}$ $\leq m+1$ for any $m$-cluster-tilting object $T \in \mathcal{E}$, as well as providing a more straightforward characterisation of the perfect modules over this endomorphism algebra.

Proposition 3.7 Let $\mathcal{E} \subseteq \mathcal{A}$ be a full, extension closed, Frobenius subcategory of an abelian category $\mathcal{A}$ such that

(i) $\mathcal{A}$ has enough projectives,

(ii) $\mathcal{E}$ contains $\operatorname{proj} \mathcal{A}$,

(iii) $\mathcal{E}$ is closed under kernels of epimorphisms, and

(iv) for any $X \in \mathcal{A}$, and any exact sequence

$$
0 \longrightarrow Y \longrightarrow P_{m} \longrightarrow \cdots \longrightarrow P_{0} \longrightarrow X \longrightarrow 0
$$

with $P_{i} \in \operatorname{proj} \mathcal{A}$, we have $Y \in \mathcal{E}$.

Let $T \in \mathcal{E}$ be $m$-cluster-tilting, and write $A=\operatorname{End}_{\mathcal{E}}(T)^{\mathrm{op}}$. Then an $A$-module $M$ is perfect if and only if it is finitely presented, and for such $M$ we have $\mathrm{p} . \operatorname{dim}_{A} M \leq m+1$. It follows that if $A$ is Noetherian, then $\mathrm{gl} \operatorname{dim} A \leq m+1$.

Proof As already noted, any perfect $A$-module is finitely presented. For the converse, let $M$ be a finitely presented $A$-module with presentation

$$
\operatorname{Hom}_{\mathcal{E}}\left(T, T_{1}\right) \stackrel{f_{*}}{\longrightarrow} \operatorname{Hom}_{\mathcal{E}}\left(T, T_{0}\right) \longrightarrow M \longrightarrow 0
$$

for some $T_{0}, T_{1} \in$ add $T$, and form the exact sequence

$$
0 \longrightarrow K_{1} \longrightarrow T_{1} \stackrel{f}{\longrightarrow} T_{0}
$$


in $\mathcal{A}$. By [25, Prop. 2.6], the subcategory $\mathcal{E}$ is contravariantly finite in $\mathcal{A}$, and hence so is add $T$. Working inductively for $1 \leq j \leq m-1$, let $r_{j}: T_{j+1} \rightarrow K_{j}$ be a right add $T$ approximation of $K_{j}$, and let $K_{j+1}$ be its kernel (in $\mathcal{A}$ ). By additionally defining $K_{0}=\operatorname{im} f$ and $K_{-1}=\operatorname{coker} f$, we obtain exact sequences

$$
0 \longrightarrow K_{j} \longrightarrow T_{j} \longrightarrow K_{j-1} \longrightarrow 0
$$

for all $0 \leq j \leq m$. Combining these to form the exact sequence

$$
0 \longrightarrow K_{m} \longrightarrow T_{m} \longrightarrow \cdots \longrightarrow T_{0} \longrightarrow K_{-1} \longrightarrow 0,
$$

we may use [25, Prop. 2.6] again to see that $K_{m} \in \mathcal{E}$.

Next we check that $K_{m} \in$ add $T$, which we do by checking that $\operatorname{Ext}_{\mathcal{E}}^{i}\left(T, K_{m}\right)=0$ for $0<i<m$. For any $j$, we may apply $\operatorname{Hom}_{\mathcal{E}}(T,-)$ to the short exact sequence

$$
0 \longrightarrow K_{j+1} \longrightarrow T_{j+1} \longrightarrow K_{j} \longrightarrow 0
$$

to find, using that $\operatorname{Ext}_{\mathcal{E}}^{i}\left(T, T_{i}\right)=0$ for $0<i<m$, that $\operatorname{Ext}_{\mathcal{E}}^{i}\left(T, K_{j+1}\right)=\operatorname{Ext}_{\mathcal{E}}^{i-1}\left(T, K_{j}\right)$ for $1<i<m$. Moreover, if $j \geq 1$, the map $T_{j+1} \rightarrow K_{j}$ in the above sequence is the

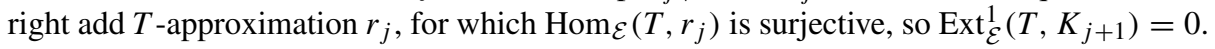
It follows that for $0<i<m$, we have

$$
\operatorname{Ext}_{\mathcal{E}}^{i}\left(T, K_{m}\right)=\operatorname{Ext}_{\mathcal{E}}^{i-1}\left(T, K_{m-1}\right)=\cdots=\operatorname{Ext}_{\mathcal{E}}^{1}\left(T, K_{m-i+1}\right)=0,
$$

as required.

It follows from the above calculations that the sequence

$$
0 \longrightarrow \operatorname{Hom}_{\mathcal{E}}\left(T, K_{j+1}\right) \longrightarrow \operatorname{Hom}_{\mathcal{E}}\left(T, T_{j+1}\right) \longrightarrow \operatorname{Hom}_{\mathcal{E}}\left(T, K_{j}\right) \longrightarrow 0
$$

is exact for all $j \geq 1$. Thus, writing $T_{m+1}=K_{m} \in \operatorname{add} T$, applying $\operatorname{Hom}_{\mathcal{E}}(T,-)$ to the sequence

$$
0 \longrightarrow T_{m+1} \longrightarrow T_{m} \longrightarrow \cdots \longrightarrow T_{1} \stackrel{f}{\longrightarrow} T_{0}
$$

yields a projective resolution of $M$ by finitely generated projective $A$-modules, and so $M$ is perfect with p. $\operatorname{dim}_{A} M \leq m+1$.

For the final statement, if $A$ is Noetherian, then every finitely generated $A$-module is also finitely presented, and thus has projective dimension at most $m+1$, as required.

We now recall some work of Iyama-Kalck-Wemyss-Yang [29], which gives a normal form for a Frobenius $m$-cluster category that is 'small enough', in the sense it admits an $m$-cluster-tilting object with Noetherian endomorphism algebra. It will follow from this description that any such Frobenius $m$-cluster category may be embedded into an abelian category in such a way that all of the assumptions of Proposition 3.7 hold. We begin with the following definitions.

Definition 3.8 An algebra $B$ is Iwanaga-Gorenstein if it is Noetherian and has finite injective dimension as both a left and right module over itself. In this case, the left and right injective dimensions coincide, and are called the Gorenstein dimension of $B$. For brevity, an IwanagaGorenstein algebra with Gorenstein dimension $d$ will be called $d$-Iwanaga-Gorenstein. If $B$ is such an algebra, we write

$$
\mathrm{GP}(B)=\left\{X \in \bmod B: \operatorname{Ext}_{B}^{i}(X, B)=0, i>0\right\}=\Omega^{d}(\bmod B)
$$


for the category of Gorenstein projective $B$-modules. This is a full, extension closed subcategory of $\bmod B$, and is a Frobenius category under the inherited exact structure [11, $\S 4.8]$. This category is sometimes $[2,28]$ denoted by $\operatorname{CM}(B)$, and the objects called maximal Cohen-Macaulay $B$-modules, but we do not do this since for certain choices of $B$ there exist definitions of Cohen-Macaulay not coinciding with this one [29, Rem. 3.3].

If $B$ is an $(m+1)$-Iwanaga-Gorenstein algebra, then the assumptions of Proposition 3.7 hold for the exact subcategory $\mathcal{E}=\operatorname{GP}(B)$ of the abelian category $\mathcal{A}=\bmod B$. Thus the endomorphism algebra of an $m$-cluster-tilting object of GP $(B)$ has global dimension at most $m+1$ whenever it is Noetherian.

Theorem 3.9 ([29, Thm. 2.7]) Let $\mathcal{E}$ be an idempotent complete Frobenius category such that $\operatorname{proj} \mathcal{E}=$ add $P$ for some $P \in \mathcal{E}$. Assume there exists $M \in \mathcal{E}$ such that $P \in$ add $M$, the endomorphism algebra $A=\operatorname{End}_{\mathcal{E}}(M)^{\mathrm{op}}$ is Noetherian, and $\mathrm{gl} . \operatorname{dim} A=d<\infty$. Then $B=\operatorname{End}_{\mathcal{E}}(P)^{\mathrm{op}}$ is Iwanaga-Gorenstein of Gorenstein dimension at most $d$, and there is an equivalence

$$
\operatorname{Hom}_{\mathcal{E}}(P,-): \mathcal{E} \stackrel{\sim}{\rightarrow} \mathrm{GP}(B)
$$

Corollary 3.10 Let $\mathcal{E}$ be a Frobenius $m$-cluster category and let $T \in \mathcal{E}$ be an $m$-clustertilting object such that $\operatorname{End}_{\mathcal{E}}(T)^{\mathrm{op}}$ is Noetherian. Let $P$ be a maximal projective summand of $T$, and write $B=\operatorname{End}_{\mathcal{E}}(P)^{\mathrm{op}}$. Then $B$ is Iwanaga-Gorenstein of Gorenstein dimension at most $m+1$, and $\mathcal{E} \simeq \mathrm{GP}(B)$.

Proof By definition, $\mathcal{E}$ is idempotent complete and gl. $\operatorname{dim} \operatorname{End}_{\mathcal{E}}(T)^{\mathrm{op}} \leq m+1$. Since $T$ is $m$-cluster-tilting, $\operatorname{proj} \mathcal{E} \subseteq$ add $T$, and so we have $\operatorname{proj} \mathcal{E}=$ add $P$. Now the result follows from Theorem 3.9.

Under the notation and assumptions of Corollary 3.10, let $e$ be the idempotent of $A=$ End $_{\mathcal{E}}(T)^{\text {op }}$ given by projection onto $P$, with respect to which $A$ is internally $(m+1)$-CalabiYau by Theorem 3.4. Then the algebra $B=\operatorname{End}_{\mathcal{E}}(P)^{\text {op }}$ is the idempotent subalgebra $e A e$. We will see below that the Gorenstein dimension of $B$ may be strictly less than $m+1$. An interesting question suggested by Corollary 3.10, to which we have no good answer at this stage, is the following: can one find reasonable conditions on an Iwanaga-Gorenstein algebra $B$ under which $\mathrm{GP}(B)$ is a Frobenius $m$-cluster category?

The remainder of the section is devoted to examples. We describe two families of examples of Frobenius cluster categories, one Hom-finite and the other Hom-infinite, to which Theorem 3.4 applies to show that the endomorphism algebras of cluster-tilting objects are internally 3-Calabi-Yau with respect to projection onto a maximal projective-injective summand. We also give a family of examples of Frobenius 1-cluster categories arising as part of the algebraic McKay correspondence.

Example 3.11 Buan-Iyama-Reiten-Scott [8] construct a family of Hom-finite stably 2Calabi-Yau Frobenius categories Sub $\Pi_{\omega}$. Here $\Pi=\Pi(\Delta)$ is the preprojective algebra associated to a graph $\Delta$, and $\omega$ is a finite product of simple reflections in the Weyl group of $\Delta$. The algebra $\Pi_{\omega}$ is a (finite dimensional) quotient of $\Pi$, and Sub $\Pi_{\omega}$ is the full subcategory of mod $\Pi_{\omega}$ given by objects isomorphic to submodules of direct sums of copies of $\Pi_{\omega}$. Then Sub $\Pi_{\omega}$ is closed under extensions and subobjects (in particular under kernels of epimorphisms), and contains $\operatorname{proj} \Pi_{\omega}$ and $\Omega\left(\bmod \Pi_{\omega}\right)$. Since Sub $\Pi_{\omega}$ is Hom-finite, it follows from Proposition 3.7 that it is a Frobenius cluster category. 
We note that the categories Sub $\Pi_{\omega}$ constructed by Buan-Iyama-Reiten-Scott contain an important class of categories considered by Geiß-Leclerc-Schröer [19], which we will also describe. For $\Delta$ a Dynkin diagram, let $\Pi$ be the preprojective algebra of type $\Delta$. If $j$ is a node of $\Delta$, write $Q_{j}$ for the injective $\Pi$-module with socle at $j$. Then for any subset $J$ of the nodes of $\Delta$, we may write $Q_{J}=\bigoplus_{j \in J} Q_{j}$, and consider the category Sub $Q_{J}$ of $\Pi$-modules isomorphic to a submodule of a direct sum of copies of $Q_{J}$, or equivalently of those $\Pi$-modules with socle supported on $J$. The category Sub $Q_{J}$ models a cluster algebra structure on the coordinate ring of a dense open subset of the partial flag variety attached to the data of $\Delta$ and $J$. For example, when $\Delta$ is of type $\mathrm{A}_{n}$ and $J$ consists of a single node, this partial flag variety is a Grassmannian. If $\omega_{0}$ is the longest word in the Weyl group of type $\Delta$, and $\omega_{0}^{K}$ is the longest word in the subgroup generated by simple reflections at nodes not in $J$, then [18, Lem. 17.2] we have

$$
\operatorname{Sub} Q_{J}=\operatorname{Sub} \Pi_{\omega_{0}^{K} \omega_{0}} .
$$

In particular, the categories $\operatorname{Sub} Q_{J}$ are Frobenius cluster categories.

If $\Pi$ is the preprojective algebra of Dynkin type $\Delta$, then we have $\bmod \Pi=\operatorname{Sub} \Pi=$ Sub $\Pi_{\omega_{0}}$, where $\omega_{0}$ is the longest word in the Weyl group of type $\Delta$, so $\bmod \Pi$ is a Frobenius cluster category. The algebra $A$ appearing in Example 2.10 is isomorphic to the endomorphism algebra of a cluster-tilting object in $\bmod \Pi$ for $\Pi$ the preprojective algebra of type $A_{2}$, and is thus internally 3-Calabi-Yau by Theorem 3.4. Similarly, the algebra $A^{\prime}$ from Example 2.10 is isomorphic to the endomorphism algebra of a cluster-tilting object in Sub $\Pi_{s_{2} s_{1} s_{3} s_{2}}=\operatorname{Sub} Q_{2}$, where $\Pi$ is the preprojective algebra of type $A_{3}$ and $Q_{2}$ is the indecomposable injective module with socle at the bivalent vertex 2 , and so $A^{\prime}$ is also internally 3-Calabi-Yau.

The category of projective objects of Sub $\Pi_{\omega}$ is given by add $\Pi_{\omega}$. Since Sub $\Pi_{\omega}$ is a Homfinite Frobenius cluster category, it follows from Corollary 3.10 that $\operatorname{Sub} \Pi_{\omega} \simeq \operatorname{GP}\left(\Pi_{\omega}\right)$. Since $\Pi_{w}$ has Gorenstein dimension at most 1 [8, Prop. III.2.2], we even have Sub $\Pi_{\omega}=$ $\operatorname{GP}\left(\Pi_{\omega}\right)$ as full subcategories of $\bmod \Pi_{w}$. Note that the Gorenstein dimension of $\Pi_{w}$ is strictly smaller than the bound provided by Corollary 3.10.

Example 3.12 Our second family of examples was introduced by Jensen-King-Su [28] to categorify the cluster algebra structure on the homogeneous coordinate ring of the Grassmannian $G_{k}^{n}$ of $k$-planes in $\mathbb{C}^{n}$. Each category in this family is of the form $\mathrm{CM}(B)$ for a Gorenstein order $B$ (depending on positive integers $1<k<n$ ) over $Z=\mathbb{C}[[t]]$. One description of $B$ is as follows. Let $\Delta$ be the graph (of affine type $\widetilde{\mathrm{A}}_{n-1}$ ) with vertex set given by the cyclic group $\mathbb{Z}_{n}$, and edges between vertices $i$ and $i+1$ for all $i$. Let $\Pi$ be the completion of the preprojective algebra on $\Delta$ with respect to the arrow ideal. Write $x$ for the sum of 'clockwise' arrows $i \rightarrow i+1$, and $y$ for the sum of 'anti-clockwise' arrows $i \rightarrow i-1$. Then we have

$$
B=\Pi /\left\langle x^{k}-y^{n-k}\right\rangle \text {. }
$$

In this description, $Z$ may be identified with the centre $\mathbb{C}[[x y]]$ of $B$.

Objects of $\operatorname{CM}(B)$ are $B$-modules that are free and finitely generated over $Z$. Since $Z$ is a principal ideal domain, and hence Noetherian, any submodule of a free and finitely generated $Z$-module is also free and finitely generated, and so $\mathrm{CM}(B)$ is closed under subobjects. In particular, $\mathrm{CM}(B)$ is closed under kernels of epimorphisms. Moreover [28, Cor. 3.7], $B \in \mathrm{CM}(B)$, and so $\Omega(\bmod B) \subseteq \mathrm{CM}(B)$.

As a $Z$-module, any object $M \in \mathrm{CM}(B)$ is isomorphic to $Z^{k}$ for some $k$, and so $\operatorname{End}_{Z}(M)^{\text {op }} \cong Z^{k^{2}}$ is a finitely generated $Z$-module. Since $Z$ is Noetherian, the algebra $\operatorname{End}_{B}(M)^{\text {op }} \subseteq \operatorname{End}_{Z}(M)^{\text {op }}$ is also finitely generated as a $Z$-module. Thus $\operatorname{End}_{B}(M)^{\text {op }}$ is Noetherian, as it is finitely generated as a module over the commutative Noetherian ring $Z$. 
We may now apply Proposition 3.7 to see that any cluster-tilting object $T \in \mathrm{CM}(B)$ satisfies gl. $\operatorname{dim} \operatorname{End}_{B}(T)^{\mathrm{op}} \leq 3$. Moreover [28, Cor. 4.6], $\underline{\mathrm{CM}}(B)=\underline{\operatorname{Sub}} Q_{k}$, where $Q_{k}$ is an indecomposable injective module for the preprojective algebra of type $A_{n-1}$ (see Example 3.11), so $\underline{\mathrm{CM}}(B)$ is 2-Calabi-Yau, and $\mathrm{CM}(B)$ is a Frobenius cluster category. This category is not Hom-finite, unlike the categories $\operatorname{Sub} \Pi_{\omega}$.

The algebra $B$ is 1-Iwanaga-Gorenstein, so the Gorenstein dimension is again strictly smaller than the bound in Corollary 3.10, and we have equalities $\operatorname{CM}(B)=\operatorname{GP}(B)=\operatorname{Sub} B$ [28, Cor. 3.7].

Baur-King-Marsh [5, Thm. 10.3] show that for certain cluster-tilting objects $T \in \mathrm{CM}(B)$, the endomorphism algebra $\operatorname{End}_{B}(T)^{\text {op }}$ is isomorphic to a frozen Jacobian algebra (Definition 5.1) associated to a dimer model on a disk, with the projection onto a maximal projective-injective summand corresponding to the sum of idempotents at the frozen vertices. By Theorem 3.4, these dimer algebras, which satisfy a natural consistency condition $[5, \S 5]$, are internally $3-C a l a b i-Y a u$ with respect to their boundary idempotent; cf. Broomhead $[7, \S 7]$, who shows that consistent dimer models on closed surfaces give rise to 3-Calabi-Yau Jacobian algebras.

Example 3.13 The algebraic McKay correspondence provides many examples of Frobenius 1 -cluster categories. Let the special linear group $\mathrm{SL}_{2}(\mathbb{C})$ act on $\mathbb{C}[[x, y]]$ in the natural way. Let $G$ be a finite subgroup of $\mathrm{SL}_{2}(\mathbb{C})$, and consider the invariant ring $R=\mathbb{C}[[x, y]]^{G}$. For example, if $G$ is cyclic of order $n$, generated by

$$
\left(\begin{array}{cc}
\omega & 0 \\
0 & \omega^{-1}
\end{array}\right)
$$

for some primitive $n$-th root of unity $\omega$, then $R=\mathbb{C}\left[\left[x^{n}, x y, y^{n}\right]\right]$.

A well-known result of Herzog [24] shows that $\mathbb{C}[[x, y]]$ is an additive generator (or equivalently, a 1-cluster-tilting object) of the Frobenius category $\mathrm{CM}(R)$ of maximal Cohen-Macaulay $R$-modules. By computing Auslander-Reiten sequences in $\operatorname{CM}(R)$, as in Leuschke-Wiegand [34, Prop. 13.22], one can see that the Auslander-Reiten translation on $\mathrm{CM}(R)$ is trivial, and so $\mathrm{CM}(R)$ is stably 1-Calabi-Yau. Let $T$ be a basic $R$-module such that $\operatorname{add}_{R} T=\operatorname{add}_{R} \mathbb{C}[[x, y]]$, so that $T$ is the unique (up to isomorphism) basic 1-cluster-tilting object of CM(R). By Auslander's Theorem [4] and a result of Reiten-Van den Bergh [39], there are isomorphisms

$$
\begin{aligned}
& \operatorname{End}_{R}(T)^{\mathrm{op}} \stackrel{\sim}{\rightarrow} \Pi(\widetilde{\Delta}), \\
& \underline{\text { End }}_{R}(T)^{\mathrm{op}} \stackrel{\sim}{\rightarrow} \Pi(\Delta),
\end{aligned}
$$

where $\widetilde{\Delta}$ is the extended Dynkin diagram given by the McKay graph of $G$, and $\Delta$ is its unextended counterpart. It is well-known that $\Pi(\Delta)$ is finite dimensional, so $\underline{\mathrm{CM}}(R)$ is Hom-finite, and that $\mathrm{gl} \operatorname{dim} \Pi(\widetilde{\Delta}) \lesseqgtr 2$. Thus $\operatorname{CM}(R)$ is a Frobenius 1-cluster category.

The algebra $\operatorname{End}_{R}(T)^{\mathrm{op}} \cong \Pi(\widetilde{\Delta})$ is bimodule 2-Calabi-Yau, which is consistent with (but stronger than) the conclusion of Theorem 3.4.

\section{From internally $d$-Calabi-Yau algebras to $d$-cluster-tilting objects}

Theorem 3.4 shows how internally $(m+1)$-Calabi-Yau algebras arise as endomorphism algebras of cluster-tilting objects in Frobenius $m$-cluster categories. In this section we work 
in the opposite direction, and show how to construct a Frobenius category admitting a $(d-1)$ cluster-tilting object from the data of an internally $d$-Calabi-Yau algebra, thus generalising a result of Amiot-Iyama-Reiten [2, Thm. 2.3] on bimodule $d$-Calabi-Yau algebras. Since we will work only with Noetherian algebras, by Corollary 3.10 we should expect to produce categories of the form GP $(B)$ for some Iwanaga-Gorenstein algebra $B$, and indeed this is what we shall do. Our main result is the following.

Theorem 4.1 (cf. [2, Thm. 2.3]) Let $A$ be a Noetherian algebra and let $e \in A$ be an idempotent such that $A /\langle e\rangle$ is finite dimensional, and both $A$ and $A^{\mathrm{op}}$ are internally $d$ Calabi-Yau with respect to $e$. Write $B=e A e$ and $\underline{A}=A /\langle e\rangle$. Then

(i) B is Iwanaga-Gorenstein with Gorenstein dimension at most d, so $\mathrm{GP}(B)$ is a Frobenius category,

(ii) eA is $(d-1)$-cluster-tilting in $\mathrm{GP}(B)$, and

(iii) there are natural isomorphisms $\operatorname{End}_{B}(e A)^{\mathrm{op}} \stackrel{\sim}{\rightarrow} A$ and $\operatorname{End}_{\mathrm{GP}(B)}(e A)^{\mathrm{op}} \stackrel{\sim}{\rightarrow} \underline{A}$.

Remark 4.2 While all of the conclusions of Theorem 4.1, except for $B$ being IwanagaGorenstein, refer only to left $B$-modules, the proof we will give uses the assumption that $A^{\text {op }}$ is internally $d$-Calabi-Yau to draw conclusions about right $A$-modules. This applies in particular to showing that the right $A$-module $e A$ is cluster-tilting in the category of Gorenstein projective $B$-modules; see Lemmas 4.8 and 4.9.

Since the assumptions of Theorem 4.1 are symmetric in $A$ and $A^{\text {op }}$, we may also conclude that $\mathrm{GP}\left(B^{\mathrm{op}}\right)$ is a Frobenius category in which $A e$ is a $(d-1)$-cluster-tilting object, and there are natural isomorphisms $\operatorname{End}_{B^{\mathrm{op}}}(A e)^{\mathrm{op}} \stackrel{\sim}{\rightarrow} A^{\mathrm{op}}$ and $\operatorname{End}_{\mathrm{GP}\left(B^{\mathrm{op}}\right)}(A e)^{\mathrm{op}} \stackrel{\sim}{\rightarrow} \underline{A}^{\mathrm{op}}$.

We emphasise two cases in which the assumptions of Theorem 4.1 may be made to appear one-sided. Firstly, as in Remark 2.3, if $A$ is a finite dimensional algebra then it is internally $d$ Calabi-Yau with respect to $e$ if and only if the same is true of $A^{\text {op }}$. Secondly, if $A$ is bimodule internally $d$-Calabi-Yau with respect to $e$, then (Remark 2.6) so is $A^{\text {op }}$, and therefore both $A$ and $A^{\text {op }}$ are internally $d$-Calabi-Yau with respect to $e$ by Corollary 2.9.

We note that Amiot-Iyama-Reiten's result [2, Thm. 2.3] is a special case of our Theorem 4.1. To obtain the same conclusions, they assume that $A$ is Noetherian, $A /\langle e\rangle$ is finite dimensional, and that $A$ is bimodule $d$-Calabi-Yau. By Corollary 2.9, this means that both $A$ and $A^{\text {op }}$ are internally $d$-Calabi-Yau with respect to any idempotent, in particular with respect to $e$.

The rest of the section is largely devoted to proving Theorem 4.1, so we let $A, e, \underline{A}$ and $B$ be as in the assumptions of this theorem. We begin with the following straightforward observation.

Proposition 4.3 The algebra B is Noetherian.

Proof Any left ideal $I$ of $B$ is of the form $e \widetilde{I}$ for a left ideal $\widetilde{I}=A I$ of $A$. So any ascending chain of left ideals of $B$ determines and is determined by such a chain of ideals of $A$, which stabilises as $A$ is Noetherian. A similar argument shows that $B$ is right Noetherian.

Proposition 4.4 (cf. [2, Lem. 2.6]) For any $X \in \bmod \underline{A}$, we have

(i) $\operatorname{Ext}_{A}^{i}(X, A)=0$ for $i \neq d$, and

(ii) $\operatorname{Ext}_{A}^{i}(X, A e)=0$ for any $i \in \mathbb{Z}$.

Proof Both $A$ and $A e$ are finitely generated projective $A$-modules, and so are in particular perfect. Since $\underline{A}$, and therefore $X$, is finite dimensional, $X$ is a finitely generated $A$-module, 
and thus perfect since $A$ is Noetherian of finite global dimension. Now we can use the internal Calabi-Yau duality of $A$ to deduce that

$$
\operatorname{Ext}_{A}^{i}(X, A)=\operatorname{DExt}_{A}^{d-i}(A, X)=0
$$

and

$$
\operatorname{Ext}_{A}^{i}(X, A e)=\mathrm{DExt}_{A}^{d-i}(A e, X)=0
$$

for $i \neq d$, since $A$ and $A e$ are projective. Since $X \in \bmod \underline{A}$, we have $e X=0$, and so

$$
\operatorname{Ext}_{A}^{d}(X, A e)=\mathrm{DHom}_{A}(A e, X)=\mathrm{D}(e X)=0 .
$$

The assumption of part (i) of Proposition 4.4 is slightly more restrictive than that $[2$, Lem. 2.6(a)]. This is necessary for the result to hold in our setting, since our $A$ is only internally $d$-Calabi-Yau. However, this stronger assumption is satisfied whenever [2, Lem. 2.6(a)] is used in the proof of [2, Thm. 2.3].

The following results (Proposition 4.5 and Lemmas 4.6, 4.8 and 4.9) are now close analogues of [2, Prop. 2.7, Lem. 2.9-2.11], with very similar proofs. For the convenience of the reader, and to make the paper more self-contained, we give a complete argument using our notation and conventions.

Proposition 4.5 (cf. [2, Prop. 2.7]) We have isomorphisms

$$
\operatorname{Ext}_{B}^{i}(e A, B) \cong \begin{cases}A e, & i=0 \\ 0, & i \neq 0\end{cases}
$$

of $A \otimes_{\mathbb{K}} B^{\mathrm{op}}$-modules, and isomorphisms

$$
\operatorname{Ext}_{B}^{i}(e A, e A) \cong \begin{cases}A^{\mathrm{op}}, & i=0, \\ 0, & 0<i<d-1,\end{cases}
$$

of vector spaces, the isomorphism in case $i=0$ being additionally an isomorphism of algebras.

Proof We can compute $\operatorname{Ext}_{B}^{i}(e A, B)$ as the cohomology of

$$
\mathbf{R H o m}_{B}(e A, B) \cong \mathbf{R H o m}_{B}\left(e A, \mathbf{R H o m}_{A}(A e, A e)\right) \cong \mathbf{R H o m}_{A}\left(A e \stackrel{\mathbf{L}}{\left.\otimes_{B} e A, A e\right),}\right.
$$

and wish to show that this is isomorphic to the cohomology of $\operatorname{RHom}_{A}(A, A e)$. To do this, we show that

$$
\mathbf{R H o m}_{A}\left(A e \stackrel{\mathbf{L}}{\otimes}{ }_{B} e A, A e\right) \cong \mathbf{R H o m}_{A}(A, A e) .
$$

Let $f$ be the composition of the natural map

$$
A e \stackrel{\mathbf{L}}{\otimes_{B}} e A \rightarrow \mathrm{H}^{0}\left(A e \stackrel{\mathbf{L}}{\otimes_{B}} e A\right)=A e \otimes_{B} e A
$$

with the multiplication map $A e \otimes_{B} e A \rightarrow A$, and let $X$ be the mapping cone of $f$, so we have a triangle

$$
A e \stackrel{\mathbf{L}}{\otimes}_{B} e A \stackrel{f}{\longrightarrow} A \longrightarrow X \longrightarrow A e \stackrel{\mathbf{L}}{\otimes}{ }_{B} e A[1]
$$


in $\mathcal{D} A$. The map $e A \otimes_{A} f$ is the natural isomorphism $B \stackrel{\mathbf{L}}{\otimes_{B}} e A \stackrel{\sim}{\rightarrow} e A$, so $e A \otimes_{A} X=0$. It follows that $e \mathrm{H}^{i}(X)=0$, and hence $\mathrm{H}^{i}(X) \in \bmod \underline{A}$ for all $i \in \mathbb{Z}$. Thus, by Proposition 4.4, $\operatorname{Ext}_{A}^{j}\left(\mathrm{H}^{i}(X), A e\right)=0$ for all $i, j \in \mathbb{Z}$.

We can compute $\mathrm{H}^{k}\left(\mathbf{R} \operatorname{Hom}_{A}(X, A e)\right)$ via a hypercohomology spectral sequence ${ }^{I I} E_{r}^{i j}$ [40, §5.7.9, see also Defn. 5.6.2], in which

$$
{ }^{I I} E_{2}^{i j}=\operatorname{Ext}_{A}^{j}\left(\mathrm{H}^{i}(X), A e\right)=0
$$

as above. It follows that $\mathrm{H}^{k}\left(\mathbf{R H o m}_{A}(X, A e)\right)=0$ for all $k$, and so $\mathbf{R H o m}_{A}(X, A e)=0$. Now applying $\mathbf{R} \operatorname{Hom}_{A}(-, A e)$ to the triangle (4.1) yields the required isomorphism

$$
\mathbf{R H o m}_{A}\left(A e \stackrel{\mathbf{L}}{\otimes}{ }_{B} e A, A e\right) \cong \mathbf{R H o m}_{A}(A, A e)
$$

in $\mathcal{D} A \otimes_{\mathbb{K}} B^{\text {op }}$, from which the first assertion follows by our initial calculations.

Similarly, we have isomorphisms

$$
\mathbf{R H o m}_{B}(e A, e A) \cong \mathbf{R H o m}_{B}\left(e A, \mathbf{R H o m}_{A}(A e, A)\right) \cong \mathbf{R H o m}_{A}\left(A e \stackrel{\mathbf{L}}{\otimes_{B}} e A, A\right),
$$

and so to obtain the second assertion we wish to show that

$$
\mathbf{R H o m}_{A}\left(A e \stackrel{\mathbf{L}}{\otimes}{ }_{B} e A, A\right) \cong \mathbf{R H o m}_{A}(A, A) .
$$

Both $A e$ and $e A$ are concentrated in degree 0 , so by triangle (4.1) we have $\mathrm{H}^{i}\left(A e \otimes_{B} \otimes_{B} A\right)=0$ for $i>0$, and so $\mathrm{H}^{i}(X)=0$ for $i>0$. Recalling that $\mathrm{H}^{i}(X) \in \bmod \underline{A}$, it follows from Proposition 4.4 that $\operatorname{Ext}_{A}^{j}\left(\mathrm{H}^{i}(X), A\right)=\operatorname{Hom}_{\mathcal{D} A}(X, A[i])=0$ for $j \neq d$. By an analogous spectral sequence argument to above, $\mathrm{H}^{i}\left(\operatorname{RHom}_{A}(X, A)\right)=0$ for $i<d$.

From (4.1), we obtain the long exact sequence

$$
\cdots \longrightarrow \operatorname{Hom}_{\mathcal{D} A}(X, A[i]) \longrightarrow \operatorname{Hom}_{\mathcal{D} A}(A, A[i]) \longrightarrow \operatorname{Hom}_{\mathcal{D} A}\left(A e^{\mathbf{L}} \otimes_{B} e A, A[i]\right) \longrightarrow \cdots
$$

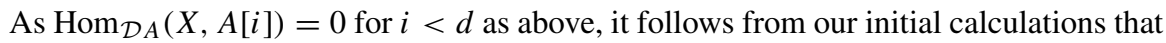

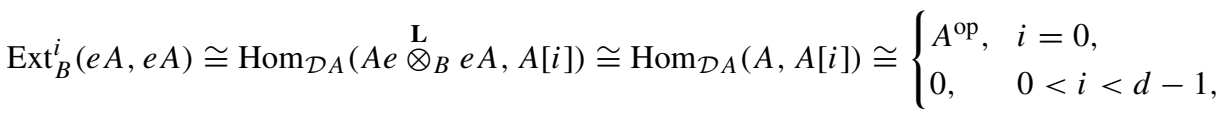
as required.

Lemma 4.6 (cf. [2, Lem. 2.9]) For any $X \in \bmod B$, we have

$$
\text { p. } \operatorname{dim}_{A^{\mathrm{op}}} \operatorname{Hom}_{B}(X, e A) \leq d-2 .
$$

Proof Since $B$ is Noetherian by Proposition 4.3, $X$ has a projective presentation $P_{1} \rightarrow P_{0} \rightarrow$ $X \rightarrow 0$ with $P_{0}$ and $P_{1}$ finitely generated. Applying $\operatorname{Hom}_{B}(-, e A)$ gives the exact sequence

$$
0 \longrightarrow \operatorname{Hom}_{B}(X, e A) \longrightarrow \operatorname{Hom}_{B}\left(P_{0}, e A\right) \longrightarrow \operatorname{Hom}_{B}\left(P_{1}, e A\right)
$$

of $A^{\mathrm{op}}$-modules. Since $\operatorname{Hom}_{B}\left(P_{i}, e A\right)$ is a projective $A^{\mathrm{op}}$-module, the above sequence shows that $\operatorname{Hom}_{B}(X, e A)$ is a second syzygy module. Since gl. $\operatorname{dim} A^{\text {op }} \leq d$ by the assumption that $A^{\text {op }}$ is internally $d$-Calabi-Yau, it follows that p. $\operatorname{dim}_{A^{\text {op }}} \operatorname{Hom}_{B}(X, e A) \leq d-2$. 
Remark 4.7 We can obtain the statement that $\mathrm{gl} \cdot \operatorname{dim} A^{\mathrm{op}} \leq d$ needed in the proof of Lemma 4.6 without assuming that $A^{\text {op }}$ is internally $d$-Calabi-Yau. By Noetherianity of $A$, we have gl. $\operatorname{dim} A^{\text {op }}=\mathrm{gl}$. $\operatorname{dim} A$, and $\mathrm{gl} . \operatorname{dim} A \leq d$ since $A$ is internally $d$-Calabi-Yau. However, the next two results, Lemmas 4.8 and 4.9 , will use this assumption on $A^{\text {op }}$ in a more fundamental way.

Lemma 4.8 (cf. [2, Lem. 2.10]) If $X \in \mathrm{GP}(B)$ and $\operatorname{Ext}_{B}^{i}(X, e A)=0$ for all $0<i<d-1$, then $X \in \operatorname{add}_{B}(e A)$.

Proof Pick an exact sequence

$$
0 \longrightarrow Y \longrightarrow P_{d-3} \longrightarrow \cdots \longrightarrow P_{0} \longrightarrow X \longrightarrow 0
$$

in which each $P_{i}$ is a finitely generated projective $B$-module. By the assumption on the vanishing of $\operatorname{Ext}_{B}^{i}(X, e A)$, we can apply $\operatorname{Hom}_{B}(-, e A)$ to obtain an exact sequence

$$
0 \rightarrow \operatorname{Hom}_{B}(X, e A) \rightarrow \operatorname{Hom}_{B}\left(P_{0}, e A\right) \rightarrow \cdots \rightarrow \operatorname{Hom}_{B}\left(P_{d-3}, e A\right) \rightarrow \operatorname{Hom}_{B}(Y, e A) \rightarrow 0
$$

of $A^{\text {op }}$-modules. Each $\operatorname{Hom}_{B}\left(P_{i}, e A\right)$ is a projective $A^{\text {op }}$-module, and by Lemma 4.6 we have p. $\operatorname{dim}_{A^{\text {op }}} \operatorname{Hom}_{B}(Y, e A) \leq d-2$, so $\operatorname{Hom}_{B}(X, e A)$ is also a projective $A^{\text {op }}$-module. It follows that $\operatorname{Hom}_{B}(X, B)=\operatorname{Hom}_{B}(X, e A) e \in \operatorname{add}_{B \text { op }}(A e)$. By [2, Prop. 1.3(b)] there are quasi-inverse dualities

$$
\begin{array}{r}
\operatorname{Hom}_{B}(-, B): \operatorname{GP}(B) \rightarrow \operatorname{GP}\left(B^{\text {op }}\right), \\
\operatorname{Hom}_{B^{\text {op }}}(-, B): \operatorname{GP}\left(B^{\text {op }}\right) \rightarrow \operatorname{GP}(B) .
\end{array}
$$

Since we are assuming $A^{\mathrm{op}}$ is also internally $d$-Calabi-Yau with respect to $e$, we can apply Proposition 4.5 to $A^{\text {op }}$ to obtain an isomorphism $\operatorname{Hom}_{B \text { op }}(A e, B) \stackrel{\sim}{\rightarrow} e A$ of $B$-modules. Therefore

$$
X \cong \operatorname{Hom}_{B^{\text {op }}}\left(\operatorname{Hom}_{B}(X, B), B\right) \in \operatorname{add}_{B}\left(\operatorname{Hom}_{B \text { op }}(A e, B)\right)=\operatorname{add}_{B}(e A)
$$

as required.

Lemma 4.9 (cf. [2, Lem. 2.11]) If $X \in \mathrm{GP}(B)$ and $\operatorname{Ext}_{B}^{i}(e A, X)=0$ for all $0<i<d-1$, then $X \in \operatorname{add}_{B}(e A)$.

Proof The quasi-inverse dualities

$$
\begin{gathered}
\operatorname{Hom}_{B}(-, B): \operatorname{GP}(B) \rightarrow \operatorname{GP}\left(B^{\text {op }}\right), \\
\operatorname{Hom}_{B^{\text {op }}}(-, B): \operatorname{GP}\left(B^{\text {op }}\right) \rightarrow \operatorname{GP}(B)
\end{gathered}
$$

from [2, Prop. 1.3(b)] preserve extension groups. Since $\operatorname{Hom}_{B}(e A, B) \cong$ Ae by Proposition 4.5, it follows that $\operatorname{Ext}_{B^{\text {op }}}^{i}\left(\operatorname{Hom}_{B}(X, B), A e\right)=0$ for all $0<i<d-1$. Thus by applying Lemma 4.8 to $A^{\text {op }}$ and $\operatorname{Hom}_{B}(X, B) \in \operatorname{GP}\left(A^{\text {op }}\right)$, we find that $\operatorname{Hom}_{B}(X, B) \in \operatorname{add}_{B}$ op $(A e)$. Then, as in Lemma 4.8, applying $\operatorname{Hom}_{B}$ op $(-, B)$ gives $X \in \operatorname{add}_{B}(e A)$.

We are now ready to prove Theorem 4.1.

Proof (i) We have already shown in Proposition 4.3 that $B$ is Noetherian, so it remains to show that $B$ has injective dimension at most $d$ on each side. First we show that $\operatorname{Ext}_{B}^{d+1}(X, B)=0$ for all $X \in \bmod B$. Given such an $X$, let $Y=A e \otimes_{B} X$, and let $\mathbf{P}$ be a projective resolution of $Y$. Then $e \mathbf{P}$ is a bounded complex in the full subcategory $\operatorname{add}(e A)$ 
of $\bmod B$, quasi-isomorphic to $e Y=X$. By Proposition 4.5, $\operatorname{Ext}_{B}^{i}(e A, B)=0$ for $i>0$, so another spectral sequence argument (now using ${ }^{I} E_{p q}^{r}$ from [40, Defn. 5.6.1]) shows that

$$
\operatorname{Ext}_{B}^{d+1}(X, B) \cong \mathrm{H}^{d+1}\left(\operatorname{Hom}_{B}(e \mathbf{P}, B)\right),
$$

where $\operatorname{Hom}_{B}(e \mathbf{P}, B)$ denotes the complex obtained by applying $\operatorname{Hom}_{B}(-, B)$ to $e \mathbf{P}$. Since

$$
\begin{aligned}
\operatorname{Hom}_{B}(e \mathbf{P}, B) & =\operatorname{Hom}_{B}\left(e A \otimes_{A} \mathbf{P}, B\right) \\
& =\operatorname{Hom}_{A}\left(\mathbf{P}, \operatorname{Hom}_{B}(e A, B)\right) \cong \operatorname{Hom}_{A}(\mathbf{P}, A e),
\end{aligned}
$$

with the final isomorphism coming from Proposition 4.5, it follows that

$$
\operatorname{Ext}_{B}^{d+1}(X, B) \cong \mathrm{H}^{d+1}\left(\operatorname{Hom}_{A}(\mathbf{P}, A e)\right) \cong \operatorname{Ext}_{A}^{d+1}(Y, A e)=0
$$

since gl. $\operatorname{dim} A \leq d$ by assumption. A dual argument, using that $A^{\text {op }}$ is internally $d$ Calabi-Yau with respect to $e$, shows that $\operatorname{Ext}_{B^{\text {op }}}^{d+1}(X, B)=0$ for all $X \in \bmod B^{\text {op }}$. It follows that $B$ is Iwanaga-Gorenstein of dimension at most $d$, and so $\operatorname{GP}(B)$ is Frobenius $[11, \S 4.8]$.

(ii) Since $A$ is Noetherian, the left ideal $\langle e\rangle=A e A$ is finitely generated. Thus it has a finite generating set contained in $e A$, which must generate $e A \subseteq A e A$ as a $B$-module, so $e A \in \bmod B$. Now $e A \in \operatorname{GP}(B)$ and $\operatorname{Ext}_{B}^{i}(e A, e A)=0$ for $0<i<d-1$ by Proposition 4.5. This, together with Lemmas 4.8 and 4.9 , shows that $e A$ is $(d-1)$ cluster-tilting in $\mathrm{GP}(B)$.

(iii) We have $\operatorname{End}_{B}(e A)^{\mathrm{op}} \cong A$ by Proposition 4.5 , and thus we have an equivalence

$$
\operatorname{Hom}_{B}(e A,-): \operatorname{add}_{B}(e A) \stackrel{\sim}{\rightarrow} \operatorname{add}_{A} A .
$$

By Proposition 4.5 again, $\operatorname{Hom}_{B}(e A, B) \cong A e$. It follows that

$$
\begin{aligned}
\operatorname{End}_{\underline{\mathrm{GP}}(B)}(e A)^{\mathrm{op}} & =\operatorname{End}_{B}(e A)^{\mathrm{op}} /\left\langle\operatorname{add}_{B} B\right\rangle \\
& \cong \operatorname{End}_{A}(A)^{\mathrm{op}} /\left\langle\operatorname{add}_{A}(A e)\right\rangle \cong A /\langle e\rangle=\underline{A}
\end{aligned}
$$

where $\langle\mathcal{C}\rangle$ denotes the ideal of maps factoring through the subcategory $\mathcal{C}$.

In the setting of Theorem 4.1, we would also like to conclude that $\operatorname{GP}(B)$ is stably $(d-1)$ Calabi-Yau. We now show, using descriptions by Kalck-Yang [30] of GP $(B)$ in terms of (complexes of) $A$-modules, that this category is $(d-1)$-Calabi-Yau when we strengthen the assumptions of Theorem 4.1 to require that $A$ is bimodule internally $d$-Calabi-Yau.

Theorem 4.10 Let $A$ be a Noetherian algebra and let $e \in A$ be an idempotent such that $A /\langle e\rangle$ is finite dimensional, and $A$ is bimodule internally $d$-Calabi-Yau with respect to $e$. Write $B=e$ Ae. Then all of the conclusions of Theorem 4.1 hold, and moreover $\mathrm{GP}(B)$ is $(d-1)$-Calabi-Yau.

Proof By Corollary 2.9, $A$ and $A^{\text {op }}$ are internally $d$-Calabi-Yau with respect to $e$, so our assumptions imply those of Theorem 4.1. It remains to check that $\underline{\mathrm{GP}}(B)$ is $(d-1)$-CalabiYau.

By [30, Prop. 2.10] (see also [16, §2-3]), there exists a dg-algebra $C$ and a dg-algebra homomorphism $A \rightarrow C$, where $A$ is considered as a dg-algebra concentrated in degree 0 , such that $C$ fits into a recollement

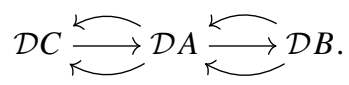


Moreover, $C^{i}=0$ for $i>0$ and $\mathrm{H}^{0}(C)=A /\langle e\rangle=\underline{A}$. Since $\underline{A}$ is finite dimensional and gl. $\operatorname{dim} A \leq d$, it follows from [30, Cor. 2.13] that $\operatorname{dim} \mathrm{H}^{i}(C)<\infty$ for all $i$, and so per $C$ is Hom-finite. The proof of this corollary also shows that $\mathcal{D}_{\mathrm{fd}}(C) \subseteq \operatorname{per} C$.

By [30, Proof of Cor. 2.12], the functor $i_{*}=\operatorname{RHom}_{C}(C,-): \mathcal{D} C \rightarrow \mathcal{D} A$ induces a triangle equivalence $\mathcal{D} C \stackrel{\sim}{\rightarrow} \mathcal{D}_{\underline{A}}(A)$, which restricts to a triangle equivalence $\mathcal{D}_{\mathrm{fd}}(C) \stackrel{\sim}{\rightarrow}$ $\mathcal{D}_{\mathrm{fd}, \underline{A}}(A)$. Thus for any $M \in \mathcal{D}_{\mathrm{fd}}(C)$ and $N \in \operatorname{per} C$, we have functorial isomorphisms

$\operatorname{DHom}_{\mathcal{D} C}(M, N)=\operatorname{DHom}_{\mathcal{D} A}\left(i_{*} M, i_{*} N\right)=\operatorname{Hom}_{\mathcal{D} A}\left(i_{*} N, i_{*} M[d]\right)=\operatorname{Hom}_{\mathcal{D} C}(N, M[d])$,

the second coming from Theorem 2.8, using the assumption that $A$ is bimodule internally $d$-Calabi-Yau with respect to $e$. Thus ( $\operatorname{per} C, \mathcal{D}_{\mathrm{fd}}(C)$, add $C$ ) is a $d$-Calabi-Yau triple in the sense of Iyama-Yang [27, §5.1], and so it follows from [27, Thm. 5.8(a)] (see also [1, §1], $[22, \S 2])$ that per $C / \mathcal{D}_{\mathrm{fd}}(C)$ is $(d-1)$-Calabi-Yau.

We complete the proof by showing that $\underline{\mathrm{GP}}(B)$ is equivalent to a full triangulated subcategory of per $C / \mathcal{D}_{\mathrm{fd}}(C)$, and so is also $(d-1)$-Calabi-Yau. Since gl. $\operatorname{dim} A \leq d,[30$, Cor. 2.12a] tells us that $i^{*}=C \stackrel{\mathrm{L}}{\otimes_{A}}$-provides a triangle equivalence between the idempotent completion of $\mathcal{D}^{b} A$ / thick $A e$, denoted by $\left(\mathcal{D}^{b} A / \text { thick } A e\right)^{\omega}$, and per $C$. Moreover, [30, Proof of Cor. 2.12] shows that $i_{*}=\operatorname{RHom}_{C}(C,-)$ induces a triangle equivalence $\mathcal{D}_{\mathrm{fd}}(C) \stackrel{\sim}{\rightarrow} \mathcal{D}_{\mathrm{fd}}, \underline{A}(A)$, and that the codomain of this equivalence coincides with thick $(\bmod \underline{A})$. Since $i^{*} i_{*} \simeq 1_{\mathcal{D} C}$, we see that $i^{*}$ restricts to an equivalence thick $(\bmod \underline{A}) \stackrel{\sim}{\rightarrow} \mathcal{D}_{\mathrm{fd}}(C)$, and so induces an equivalence

$$
\frac{\left(\mathcal{D}^{b} A / \text { thick } A e\right)^{\omega}}{q(\operatorname{thick}(\bmod \underline{A}))} \stackrel{\sim}{\rightarrow} \frac{\operatorname{per} C}{\mathcal{D}_{\mathrm{fd}}(C)},
$$

where $q$ denotes the projection $\mathcal{D}^{b} A \rightarrow \mathcal{D}^{b} A$ / thick $A e$, which restricts to an equivalence on thick $(\bmod \underline{A})$ by the above observations. We also have equivalences

$$
\frac{\mathcal{D}^{b} A / \text { thick } A e}{q(\text { thick }(\bmod \underline{A}))} \stackrel{\sim}{\rightarrow} \mathcal{D}^{b} B / \operatorname{per} B \stackrel{\sim}{\rightarrow} \underline{\mathrm{GP}}(B),
$$

the first from [30, Prop. 3.3] and the second from a result of Buchweitz [11, Thm. 4.4.1]. Since $\mathcal{D}^{b} A$ / thick $A e$ is a full triangulated subcategory of its idempotent completion, combining the above equivalences gives a triangle equivalence of $\mathrm{GP}(B)$ with a full triangulated subcategory of the $(d-1)$-Calabi-Yau triangulated category per $C / \mathcal{D}_{\mathrm{fd}}(C)$, from which it follows that $\underline{\mathrm{GP}}(B)$ is itself $(d-1)$-Calabi-Yau.

It was necessary in the proof of Theorem 4.10 to use the bimodule internal Calabi-Yau symmetry of $A$ to obtain a duality between spaces of maps of complexes of $A$-modules, so it is unclear whether the conclusion that $\underline{\operatorname{GP}}(B)$ is $(d-1)$-Calabi-Yau might hold only under the weaker assumptions of Theorem 4.1. As already stated, we do not currently have any examples of internally Calabi-Yau algebras that are not bimodule internally Calabi-Yau, but it seems unlikely that the two classes coincide.

Under the assumptions of Theorem 4.10, we would like to be able to conclude that the Frobenius category $\operatorname{GP}(B)$ is in fact a Frobenius $(d-1)$-cluster category in the sense of Definition 3.3. It remains to check that gl. $\operatorname{dim} \operatorname{End}_{B}(T)^{\text {op }} \leq d$ for any $(d-1)$-clustertilting object $T \in \mathrm{GP}(B)$; a priori, we only know this for the $(d-1)$-cluster-tilting object $e A$. Whenever $\operatorname{End}_{B}(T)^{\text {op }}$ is Noetherian, we can apply Proposition 3.7 to get the desired conclusion. While this Noetherianity is automatic in some situations, such as if $B$ is finite dimensional over $\mathbb{K}$, in general it appears to be a more subtle issue. 
Remark 4.11 As indicated in the introduction, Theorems 4.1 and 4.10 are motivated by the problem of constructing Frobenius categories modelling cluster algebras. Given the seed of a cluster algebra with frozen variables, we can look for an algebra $A$ with the same quiver (up to the addition of arrows between frozen vertices), satisfying the conditions of Theorem 4.10 for $d=3$, and then apply this theorem to obtain the Frobenius category $\operatorname{GP}(B)$. Since constructing such an $A$ can be very difficult, we wish to comment on the degree to which the conditions we are imposing are necessary.

Firstly, we consider it likely that the condition that $A$ is Noetherian can be dropped, up to finding an appropriate replacement for the category $\operatorname{GP}(B)$. While $B$ may not be Noetherian if $A$ fails to be, it will still have injective dimension at most $d$ on each side, so there should be a 'good' theory of Gorenstein projective modules over $B$. For our methods to work, we would need the analogues of [2, Prop. 1.3] to hold in this setting. We would also hope for a more general version of the Iyama-Kalck-Wemyss-Yang equivalence stated here as Theorem 3.9, without the Noetherianity assumption, which would then apply to arbitrary Frobenius $m$ cluster categories, and Buchweitz's description of the stable category [11, Thm. 4.4.1].

The other conditions are more essential; if $A=\operatorname{End}_{\mathcal{E}}(T)^{\text {op }}$ for $T$ a cluster-tilting object in a Frobenius cluster category $\mathcal{E}$, and $e$ is the idempotent given by projecting onto a maximal projective summand of $T$, then $A /\langle e\rangle$ must be finite dimensional since $\underline{\mathcal{E}}$ is Hom-finite, and $A$ is internally 3-Calabi-Yau with respect to $e$ by Theorem 3.4. On the other hand, it may not be necessary for $A$ to be bimodule internally 3-Calabi-Yau.

\section{A bimodule complex for frozen Jacobian algebras}

Given a Frobenius cluster category $\mathcal{E}$ and a cluster-tilting object $T \in \mathcal{E}$, it is often the case that $A=\operatorname{End}_{\mathcal{E}}(T)^{\text {op }}$ takes the form of a frozen Jacobian algebra (see Definition 5.1 below). Indeed, this is the case for at least some cluster-tilting objects in the families of Frobenius cluster categories we described in Examples 3.11 and 3.12; see [9, Thm. 6.6], [5, Thm. 10.3]. Thus these algebras, which also come with a preferred 'frozen' idempotent, are ideal candidates for constructing stably 2-Calabi-Yau Frobenius categories via the methods of Theorems 4.1 and 4.10. Moreover, 3-Calabi-Yau properties of ordinary Jacobian algebras have been widely studied, for example by Bocklandt [6] and, in the context of dimer models on closed surfaces, by Broomhead [7].

With this in mind, the main result of this section, Theorem 5.6, shows that a frozen Jacobian algebra admitting a particular bimodule resolution (analogous to one defined by Ginzburg $[20,5.1 .5])$ is bimodule internally 3-Calabi-Yau with respect to its frozen idempotent.

Definition 5.1 ( $c f$. [9, Defn. 1.1], [15, §2.1], [17, §6.1]) An ice quiver $(Q, F)$ consists of a finite quiver $Q$ without loops and a (not necessarily full) subquiver $F$ of $Q$. Denote by $\widehat{\mathbb{K} Q}$ the completion of the path algebra of $Q$ over $\mathbb{K}$ with respect to the arrow ideal. A potential on $Q$ is a linear combination $W$ of cycles of $Q$. A vertex or arrow of $Q$ is called frozen if it is a vertex or arrow of $F$, and mutable or unfrozen otherwise. For brevity, we write $Q_{0}^{\mathrm{m}}=Q_{0} \backslash F_{0}$ and $Q_{1}^{\mathrm{m}}=Q_{1} \backslash F_{1}$ for the sets of mutable vertices and unfrozen arrows respectively. For $\alpha \in Q_{1}$ and $\alpha_{n} \cdots \alpha_{1}$ a cycle in $Q$, write

$$
\partial_{\alpha} \alpha_{n} \cdots \alpha_{1}=\sum_{\alpha_{i}=\alpha} \alpha_{i-1} \cdots \alpha_{1} \alpha_{n} \cdots \alpha_{i+1}
$$

and extend linearly. The ideal $\left\langle\partial_{\alpha} W: \alpha \in Q_{1}^{\mathrm{m}}\right\rangle$ of $\widehat{\mathbb{K} Q}$ is called the Jacobian ideal, and we may take its closure $\overline{\left\langle\partial_{\alpha} W: \alpha \in Q_{1}^{\mathrm{m}}\right\rangle}$ since $\widehat{\mathbb{K} Q}$ is a topological algebra. We define the 
frozen Jacobian algebra associated to $(Q, F, W)$ by

$$
\mathcal{J}(Q, F, W)=\widehat{\mathbb{K} Q} / \overline{\left\langle\partial_{\alpha} W: \alpha \in Q_{1}^{\mathrm{m}}\right\rangle} .
$$

Write $A=\mathcal{J}(Q, F, W)$. The above presentation of $A$ suggests a preferred idempotent $e=\sum_{v \in F_{0}} e_{v}$, which we call the frozen idempotent. We will call $B=e A e$ the boundary algebra of $A$.

Remark 5.2 If $F=\varnothing$, then $\mathcal{J}(Q, \varnothing, W)=: \mathcal{J}(Q, W)$ is the usual Jacobian algebra.

Example 5.3 Consider the ice quiver with potential $(Q, F, W)$, where

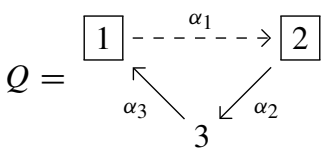

the frozen subquiver $F$ is the full subquiver on vertices 1 and 2, indicated by boxed vertices and a dashed arrow, and $W=\alpha_{3} \alpha_{2} \alpha_{1}$. Then the frozen Jacobian algebra $\mathcal{J}(Q, F, W)$ is the quotient of $\widehat{\mathbb{K} Q}$ by the relations $\partial_{\alpha_{2}} W=\alpha_{1} \alpha_{3}$ and $\partial_{\alpha_{3}} W=\alpha_{2} \alpha_{1}$, so $\mathcal{J}(Q, F, W)$ is the algebra $A$ from Example 2.10. (In this case $A$ is finite dimensional, so it agrees with the quotient of the ordinary path algebra $\mathbb{K} Q$ by the same relations.) It will follow from Theorem 5.6 below that $\mathcal{J}(Q, F, W)$ is bimodule internally 3-Calabi-Yau with respect to the idempotent $e_{1}+e_{2}$ given by summing the idempotents corresponding to frozen vertices. The usual Jacobian algebra $\mathcal{J}(Q, W)$ has the additional relation $\alpha_{3} \alpha_{2}=0$ and is not bimodule 3-Calabi-Yau; indeed, it has infinite global dimension.

Given a quiver with potential $(Q, W)$, Ginzburg [20, 5.1.5] (see also [7, §7]) defines a complex of projective bimodules over the associated Jacobian algebra. For $(Q, W)$ a quiver with potential determined by a dimer model on a torus, Broomhead shows in [7, Thm. 7.7] that if the dimer model is consistent, then this complex is isomorphic to $A=\mathcal{J}(Q, W)$ in $\mathcal{D}^{b} A^{\varepsilon}$, and thus provides a projective bimodule resolution of $A$. It follows in this case that $A$ is 3-Calabi-Yau, with this property arising from a natural symmetry in the bimodule resolution.

We will now define an analogous complex $\mathbf{P}(A)$ for a frozen Jacobian algebra $A=$ $\mathcal{J}(Q, F, W)$. Our main result (Theorem 5.6) will be that if $\mathbf{P}(A)$ is isomorphic to $A$ in $\mathcal{D}^{b} A^{\varepsilon}$, then $A$ is bimodule internally 3-Calabi-Yau with respect to the frozen idempotent $e=\sum_{v \in F_{0}} e_{v}$, in the sense of Definition 2.4. While we will write $\mathbf{P}(A)$ for this complex in order to save space, the definition depends not only on $A$ but on the ice quiver with potential $(Q, F, W)$ giving the presentation of $A$ as $\mathcal{J}(Q, F, W)$.

Recall that we write $Q_{0}^{\mathrm{m}}=Q_{0} \backslash F_{0}$ for the set of mutable vertices and $Q_{1}^{\mathrm{m}}=Q_{1} \backslash F_{1}$ for the set of unfrozen arrows. We also write $v^{+}$for the set of arrows with tail at $v$, and $v^{-}$for the set of arrows with head at $v$. Denote the arrow ideal of $A$ by $\mathfrak{m}(A)$, and let $S=A / \mathfrak{m}(A)$. For the remainder of this section, we write $\otimes=\otimes_{S}$.

Introduce formal symbols $\rho_{\alpha}$ for each $\alpha \in Q_{1}$ and $\omega_{v}$ for each $v \in Q_{0}$, and define $S$-bimodule structures on the vector spaces

$$
\begin{array}{lll}
\mathbb{K} Q_{0}=\bigoplus_{v \in Q_{0}} \mathbb{K} e_{v}, & \mathbb{K} Q_{0}^{\mathrm{m}}=\bigoplus_{v \in Q_{0}^{\mathrm{m}}} \mathbb{K} e_{v}, & \mathbb{K} F_{0}=\bigoplus_{v \in F_{0}} \mathbb{K} e_{v}, \\
\mathbb{K} Q_{1}=\bigoplus_{\alpha \in Q_{1}} \mathbb{K} \alpha, & \mathbb{K} Q_{1}^{\mathrm{m}}=\bigoplus_{\alpha \in Q_{1}^{\mathrm{m}}} \mathbb{K} \alpha, & \mathbb{K} F_{1}=\bigoplus_{\alpha \in F_{1}} \mathbb{K} \alpha,
\end{array}
$$




$$
\begin{array}{lll}
\mathbb{K} Q_{2}=\bigoplus_{\alpha \in Q_{1}} \mathbb{K} \rho_{\alpha}, & \mathbb{K} Q_{2}^{\mathrm{m}}=\bigoplus_{\alpha \in Q_{1}^{\mathrm{m}}} \mathbb{K} \rho_{\alpha}, & \mathbb{K} F_{2}=\bigoplus_{\alpha \in F_{1}} \mathbb{K} \rho_{\alpha}, \\
\mathbb{K} Q_{3}=\bigoplus_{v \in Q_{0}} \mathbb{K} \omega_{v}, & \mathbb{K} Q_{3}^{\mathrm{m}}=\bigoplus_{v \in Q_{0}^{\mathrm{m}}} \mathbb{K} \omega_{v}, & \mathbb{K} F_{3}=\bigoplus_{v \in F_{0}} \mathbb{K} \omega_{v},
\end{array}
$$

via the formulae

$$
\begin{aligned}
e_{v} \cdot e_{v} \cdot e_{v} & =e_{v}, \\
e_{h \alpha} \cdot \alpha \cdot e_{t \alpha} & =\alpha, \\
e_{t \alpha} \cdot \rho_{\alpha} \cdot e_{h \alpha} & =\rho_{\alpha}, \\
e_{v} \cdot \omega_{v} \cdot e_{v} & =\omega_{v},
\end{aligned}
$$

where $h \alpha$ and $t \alpha$ denote the head and tail of the arrow $\alpha$. For each $i$, the $S$-bimodule $\mathbb{K} Q_{i}$ splits as the direct sum

$$
\mathbb{K} Q_{i}=\mathbb{K} Q_{i}^{\mathrm{m}} \oplus \mathbb{K} F_{i} .
$$

Since $\mathbb{K} Q_{0} \cong S$, the $A$-bimodule $A \otimes \mathbb{K} Q_{0} \otimes A$ is canonically isomorphic to $A \otimes A$, and we will use the two descriptions interchangeably.

We define maps $\bar{\mu}_{i}: A \otimes \mathbb{K} Q_{i} \otimes A \rightarrow A \otimes \mathbb{K} Q_{i-1} \otimes A$ for $1 \leq i \leq 3$. The map $\bar{\mu}_{1}$ is defined by

$$
\bar{\mu}_{1}(x \otimes \alpha \otimes y)=x \otimes e_{h \alpha} \otimes \alpha y-x \alpha \otimes e_{t \alpha} \otimes y,
$$

or, composing with the natural isomorphism $A \otimes \mathbb{K} Q_{0} \otimes A \stackrel{\sim}{\rightarrow} A \otimes A$, by

$$
\bar{\mu}_{1}(x \otimes \alpha \otimes y)=x \otimes \alpha y-x \alpha \otimes y .
$$

For any path $p=\alpha_{m} \cdots \alpha_{1}$ of $Q$, we may define

$$
\Delta_{\alpha}(p)=\sum_{\alpha_{i}=\alpha} \alpha_{m} \cdots \alpha_{i+1} \otimes \alpha_{i} \otimes \alpha_{i-1} \cdots \alpha_{1},
$$

and extend by linearity to obtain a map $\Delta_{\alpha}: \widehat{\mathbb{K} Q} \rightarrow A \otimes \mathbb{K} Q_{1} \otimes A$. We then define

$$
\bar{\mu}_{2}\left(x \otimes \rho_{\alpha} \otimes y\right)=\sum_{\beta \in Q_{1}} x \Delta_{\beta}\left(\partial_{\alpha} W\right) y .
$$

Finally, let

$$
\bar{\mu}_{3}\left(x \otimes \omega_{v} \otimes y\right)=\sum_{\alpha \in v^{+}} x \otimes \rho_{\alpha} \otimes \alpha y-\sum_{\beta \in v^{-}} x \beta \otimes \rho_{\beta} \otimes y .
$$

Definition 5.4 For $A=\mathcal{J}(Q, F, W)$, let $\mathbf{P}(A)$ be the sequence

$A \otimes \mathbb{K} Q_{3}^{\mathrm{m}} \otimes A \stackrel{\mu_{3}}{\longrightarrow} A \otimes \mathbb{K} Q_{2}^{\mathrm{m}} \otimes A \stackrel{\mu_{2}}{\longrightarrow} A \otimes \mathbb{K} Q_{1} \otimes A \stackrel{\mu_{1}}{\longrightarrow} A \otimes \mathbb{K} Q_{0} \otimes A$

of $A$-bimodules, where $\mu_{1}=\bar{\mu}_{1}$, and the maps $\mu_{2}$ and $\mu_{3}$ are obtained by restricting $\bar{\mu}_{2}$ and $\bar{\mu}_{3}$ to $A \otimes \mathbb{K} Q_{2}^{\mathrm{m}} \otimes A$ and $A \otimes \mathbb{K} Q_{3}^{\mathrm{m}} \otimes A$ respectively. As $v^{+} \cup v^{-} \subseteq Q_{1}^{\mathrm{m}}$ for any $v \in Q_{0}^{\mathrm{m}}$, the map $\mu_{3}$ takes values in $A \otimes \mathbb{K} Q_{2}^{\mathrm{m}} \otimes A$ as claimed.

If $F=\varnothing$, then $\mathbf{P}(A)$ is the complex associated to $(Q, W)$ by Ginzburg [20, 5.1.5] and Broomhead [7, §7]. In the general case, $\mathbf{P}(A)$ has already appeared in work of Amiot-ReitenTodorov [3, Proof of Prop. 2.2]. 
Lemma 5.5 For a frozen Jacobian algebra $A=\mathcal{J}(Q, F, W)$, the sequence $\mathbf{P}(A)$ in Definition 5.4 is a complex of finitely generated projective A-bimodules, and there is a morphism

$A \otimes \mathbb{K} Q_{3}^{\mathrm{m}} \otimes A \stackrel{\mu_{3}}{\longrightarrow} A \otimes \mathbb{K} Q_{2}^{\mathrm{m}} \otimes A \stackrel{\mu_{2}}{\longrightarrow} A \otimes \mathbb{K} Q_{1} \otimes A \stackrel{\mu_{1}}{\longrightarrow} A \otimes A$

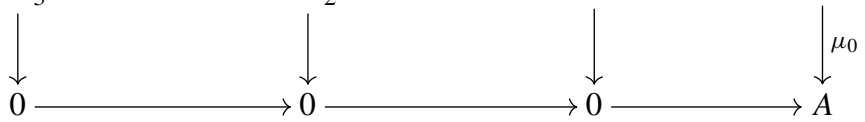

from $\mathbf{P}(A)$ to $A$, where $\mu_{0}: A \otimes A \rightarrow A$ is the multiplication in A. Moreover, the complex $0 \rightarrow \mathbf{P}(A) \rightarrow A \rightarrow 0$ is exact at $A, A \otimes A$ and $A \otimes \mathbb{K} Q_{1} \otimes A$.

Proof Each term of $\mathbf{P}(A)$ is a projective $A$-bimodule since $A$ is a projective $A$-module on each side, and is finitely generated by finiteness of $Q$. By standard results on presentations of algebras, see for example Butler-King $[12,1.2], \mu_{0}$ is surjective, im $\mu_{1}=\operatorname{ker} \mu_{0}$ and $\operatorname{im} \mu_{2}=\operatorname{ker} \mu_{1}$. Thus the only statement left to check is that $\mu_{2} \circ \mu_{3}=0$.

Let $v \in Q_{0}$, and write

$$
W_{v}=\sum_{\alpha \in v^{+}}\left(\partial_{\alpha} W\right) \alpha=\sum_{\beta \in v^{-}} \beta\left(\partial_{\beta} W\right) .
$$

We can calculate $\sum_{\gamma \in Q_{1}} \Delta_{\gamma}\left(W_{v}\right)$ using each of the two expressions, to get

$$
\begin{aligned}
& \sum_{\gamma \in Q_{1}} \Delta_{\gamma}\left(W_{v}\right)=\sum_{\alpha \in v^{+}} \sum_{\gamma \in Q_{1}} \Delta_{\gamma}\left(\partial_{\alpha} W\right) \alpha+\sum_{\alpha \in v^{+}} \partial_{\alpha} W \otimes \alpha \otimes 1, \\
& \sum_{\gamma \in Q_{1}} \Delta_{\gamma}\left(W_{v}\right)=\sum_{\beta \in v^{-}} \sum_{\gamma \in Q_{1}} \beta \Delta_{\gamma}\left(\partial_{\beta} W\right)+\sum_{\beta \in v^{-}} 1 \otimes \beta \otimes \partial_{\beta} W .
\end{aligned}
$$

If $v \in Q_{0}^{\mathrm{m}}$, then all arrows incident with $v$ are unfrozen, and so $\partial_{\alpha} W=0=\partial_{\beta} W$ in $A$ for any $\alpha \in v^{+}$and $\beta \in v^{-}$. Thus in this case we have

$$
\sum_{\alpha \in v^{+}} \sum_{\gamma \in Q_{1}} \Delta_{\gamma}\left(\partial_{\alpha} W\right) \alpha=\sum_{\gamma \in Q_{1}} \Delta_{\gamma}\left(W_{v}\right)=\sum_{\beta \in v^{-}} \sum_{\gamma \in Q_{1}} \beta \Delta_{\gamma}\left(\partial_{\beta} W\right) .
$$

It follows that

$$
\begin{aligned}
\mu_{2}\left(\mu_{3}\left(1 \otimes \omega_{v} \otimes 1\right)\right) & =\mu_{2}\left(\sum_{\alpha \in v^{+}} 1 \otimes \rho_{\alpha} \otimes \alpha-\sum_{\beta \in v^{-}} \beta \otimes \rho_{\beta} \otimes 1\right) \\
& =\sum_{\alpha \in v^{+}} \sum_{\gamma \in Q_{1}} \Delta_{\gamma}\left(\partial_{\alpha} W\right) \alpha-\sum_{\beta \in v^{-}} \sum_{\gamma \in Q_{1}} \beta \Delta_{\gamma}\left(\partial_{\beta} W\right) \\
& =0 .
\end{aligned}
$$

This completes the proof.

If the map

$A \otimes \mathbb{K} Q_{3}^{\mathrm{m}} \otimes A \stackrel{\mu_{3}}{\longrightarrow} A \otimes \mathbb{K} Q_{2}^{\mathrm{m}} \otimes A \stackrel{\mu_{2}}{\longrightarrow} A \otimes \mathbb{K} Q_{1} \otimes A \stackrel{\mu_{1}}{\longrightarrow} A \otimes A$

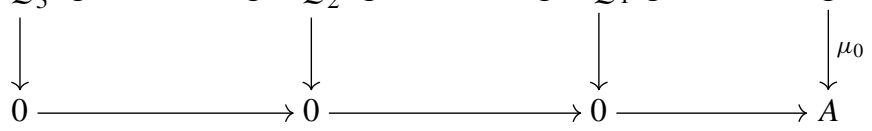

from Lemma 5.5 is a quasi-isomorphism, then $\mathbf{P}(A)$ is a projective bimodule resolution of $A$. This means that, for the presentation of $A$ as a frozen Jacobian algebra, with relations 
given by certain derivatives of the superpotential, the first syzygies are dual to the mutable vertices, and there are no higher syzygies. In particular, gl. $\operatorname{dim} A \leq 3$. By Lemma 5.5, this map is a quasi-isomorphism if and only if the cohomology of $\mathbf{P}(A)$ vanishes at $A \otimes \mathbb{K} Q_{2}^{\mathrm{m}} \otimes A$ and $A \otimes \mathbb{K} Q_{3}^{\mathrm{m}} \otimes A$ (cf. [7, Rem. 7.4]). We will usually abuse notation and denote the map $\mathbf{P}(A) \rightarrow A$ from Lemma 5.5 by $\mu_{0}$.

If $F=\varnothing$, the map $\mu_{0}: \mathbf{P}(A) \rightarrow A$ being a quasi-isomorphism implies that $A$ is 3-CalabiYau [7, Thm. 7.7] and [20, Cor. 5.3.3]. We now show that, in the general case, $\mu_{0}$ being a quasi-isomorphism implies that $A$ is bimodule internally 3-Calabi-Yau with respect to $e$.

Theorem 5.6 If $A$ is a frozen Jacobian algebra such that $\mu_{0}: \mathbf{P}(A) \rightarrow A$ is a quasiisomorphism, then $A$ is bimodule internally 3-Calabi-Yau with respect to the frozen idempotent $e=\sum_{v \in F_{0}} e_{v}$.

Proof Since $\mathbf{P}(A) \in$ per $A^{\varepsilon}$, the quasi-isomorphism $\mu_{0}: \mathbf{P}(A) \stackrel{\sim}{\rightarrow} A$ makes $\mathbf{P}(A)$ into a projective resolution of $A$, implying immediately that p. $\operatorname{dim}_{A^{\varepsilon}} A \leq 3$ and $A \in \operatorname{per} A^{\varepsilon}$. It remains to check condition (iii) from Definition 2.4.

We begin by describing $\Omega_{A}=\operatorname{RHom}_{A^{\varepsilon}}\left(A, A^{\varepsilon}\right) \in \mathcal{D}^{b} A^{\varepsilon}$. Denoting $\operatorname{Hom}_{A^{\varepsilon}}(-,-)$ by $(-,-)$, the complex $\Omega_{A}$ is given by

$$
\left(A \otimes A, A^{\varepsilon}\right) \stackrel{-\mu_{1}^{*}}{\longrightarrow}\left(A \otimes \mathbb{K} Q_{1} \otimes A, A^{\varepsilon}\right) \stackrel{\mu_{2}^{*}}{\longrightarrow}\left(A \otimes \mathbb{K} Q_{2}^{\mathrm{m}} \otimes A, A^{\varepsilon}\right) \stackrel{-\mu_{3}^{*}}{\longrightarrow}\left(A \otimes \mathbb{K} Q_{3}^{\mathrm{m}} \otimes A, A^{\varepsilon}\right)
$$

with $\mu_{i}^{*}: f \mapsto f \circ \mu_{i}$; see Keller [31, §2.7] for the signs on the differentials.

There are $A$-bimodule isomorphisms $A \otimes A \cong \bigoplus_{v \in Q_{0}} A e_{v} \otimes_{\mathbb{K}} e_{v} A$ and $A^{\varepsilon} \cong A \otimes_{\mathbb{K}} A$. Introducing the shorthand notation

$$
\mathbf{x} \otimes \mathbf{y}=\sum_{i=1}^{k} x^{i} \otimes y^{i}
$$

for elements of $A \otimes_{\mathbb{K}} A$, a homomorphism $f_{0}: A \otimes A \rightarrow A^{\varepsilon}$ is uniquely determined by the values

$$
f_{0}\left(1 \otimes e_{v} \otimes 1\right)=\mathbf{x}_{v} \otimes \mathbf{y}_{v}
$$

for each $v \in Q_{0}$. Since $1 \otimes e_{v} \otimes 1=e_{v} \otimes e_{v} \otimes e_{v}$, we must have

$$
\mathbf{x}_{v} \otimes \mathbf{y}_{v}=e_{v} \mathbf{x}_{v} \otimes \mathbf{y}_{v} e_{v} \in e_{v} A \otimes_{\mathbb{K}} A e_{v},
$$

but $\mathbf{x}_{v}$ and $\mathbf{y}_{v}$ may otherwise be chosen freely. If follows that we have an isomorphism

$$
\left(A \otimes A, A^{\varepsilon}\right) \stackrel{\sim}{\rightarrow} A \otimes \mathbb{K} Q_{3} \otimes A, \quad \quad f_{0} \mapsto \sum_{v \in Q_{0}} \mathbf{y}_{v} \otimes \omega_{v} \otimes \mathbf{x}_{v}
$$

of $A$-bimodules. Similar arguments yield explicit isomorphisms

$$
\begin{array}{ll}
\left(A \otimes \mathbb{K} Q_{1} \otimes A, A^{\varepsilon}\right) \stackrel{\sim}{\rightarrow} A \otimes \mathbb{K} Q_{2} \otimes A, & f_{1} \mapsto \sum_{\alpha \in Q_{1}} \mathbf{y}_{\alpha} \otimes \rho_{\alpha} \otimes \mathbf{x}_{\alpha}, \\
\left(A \otimes \mathbb{K} Q_{2}^{\mathrm{m}} \otimes A, A^{\varepsilon}\right) \stackrel{\sim}{\rightarrow} A \otimes \mathbb{K} Q_{1}^{\mathrm{m}} \otimes A, & f_{2} \mapsto \sum_{\alpha \in Q_{1}^{\mathrm{m}}} \mathbf{y}_{\alpha}^{\prime} \otimes \alpha \otimes \mathbf{x}_{\alpha}^{\prime}, \\
\left(A \otimes \mathbb{K} Q_{3}^{\mathrm{m}} \otimes A, A^{\varepsilon}\right) \stackrel{\sim}{\rightarrow} A \otimes \mathbb{K} Q_{0}^{\mathrm{m}} \otimes A, & f_{3} \mapsto \sum_{v \in Q_{0}^{\mathrm{m}}} \mathbf{y}_{v}^{\prime} \otimes e_{v} \otimes \mathbf{x}_{v}^{\prime},
\end{array}
$$


where the functions $f_{1}, f_{2}$ and $f_{3}$ are uniquely determined by the values

$$
\begin{aligned}
f_{1}(1 \otimes \alpha \otimes 1) & =\mathbf{x}_{\alpha} \otimes \mathbf{y}_{\alpha} \in e_{h \alpha} A \otimes_{\mathbb{K}} A e_{t \alpha}, \\
f_{2}\left(1 \otimes \rho_{\alpha} \otimes 1\right) & =\mathbf{x}_{\alpha}^{\prime} \otimes \mathbf{y}_{\alpha}^{\prime} \in e_{t \alpha} A \otimes_{\mathbb{K}} A e_{h \alpha}, \\
f_{3}\left(1 \otimes \omega_{v} \otimes 1\right) & =\mathbf{x}_{v}^{\prime} \otimes \mathbf{y}_{v}^{\prime} \in e_{v} A \otimes_{\mathbb{K}} A e_{v} .
\end{aligned}
$$

Since $\alpha \in F_{1}$ implies that $h \alpha, t \alpha \in F_{0}$, the map $\bar{\mu}_{1}: A \otimes \mathbb{K} Q_{1} \otimes A \rightarrow A \otimes \mathbb{K} Q_{0} \otimes A$ restricts to a map $A \otimes \mathbb{K} F_{1} \otimes A \rightarrow A \otimes \mathbb{K} F_{0} \otimes A$, and thus taking quotients yields a map $\mu_{1}^{\vee}: A \otimes \mathbb{K} Q_{1}^{\mathrm{m}} \otimes A \rightarrow A \otimes \mathbb{K} Q_{0}^{\mathrm{m}} \otimes A$. Explicitly, $\mu_{1}^{\vee}$ is given by

$$
\mu_{1}^{\vee}(1 \otimes \alpha \otimes 1)=1 \otimes(1-e) \alpha-\alpha(1-e) \otimes 1 .
$$

Define $\mu_{2}^{\vee}$ to be the composition of $\bar{\mu}_{2}$ with the projection $A \otimes \mathbb{K} Q_{1} \otimes A \rightarrow A \otimes \mathbb{K} Q_{1}^{\mathrm{m}} \otimes A$; explicitly

$$
\mu_{2}^{\vee}\left(1 \otimes \rho_{\alpha} \otimes 1\right)=\sum_{\beta \in Q_{1}^{\mathrm{m}}} \Delta_{\beta}\left(\partial_{\alpha} W\right) .
$$

Finally, let $\mu_{3}^{\vee}=\bar{\mu}_{3}$. Then one can check that the isomorphisms of $A$-bimodules defined above induce an isomorphism of $\Omega_{A}$ with the complex

$$
A \otimes \mathbb{K} Q_{3} \otimes A \stackrel{\mu_{3}^{\vee}}{\longrightarrow} A \otimes \mathbb{K} Q_{2} \otimes A \stackrel{\mu_{2}^{\vee}}{\longrightarrow} A \otimes \mathbb{K} Q_{1}^{\mathrm{m}} \otimes A \stackrel{\mu_{1}^{\vee}}{\longrightarrow} A \otimes \mathbb{K} Q_{0}^{\mathrm{m}} \otimes A .
$$

As an example to illustrate the necessary calculations, we show that we get an isomorphism of $\mu_{2}^{*}:\left(A \otimes \mathbb{K} Q_{1} \otimes A, A^{\varepsilon}\right) \rightarrow\left(A \otimes \mathbb{K} Q_{2}^{\mathrm{m}} \otimes A, A^{\varepsilon}\right)$ with $\mu_{2}^{\vee}: A \otimes \mathbb{K} Q_{2} \otimes A \rightarrow A \otimes \mathbb{K} Q_{1}^{\mathrm{m}} \otimes A$. It suffices to check this on the generators $1 \otimes \rho_{\alpha} \otimes 1$ of $A \otimes \mathbb{K} Q_{2} \otimes A$. First observe that under the isomorphism $\left(A \otimes \mathbb{K} Q_{1} \otimes A, A^{\varepsilon}\right) \stackrel{\sim}{\rightarrow} A \otimes \mathbb{K} Q_{2} \otimes A$, the preimage of $1 \otimes \rho_{\alpha} \otimes 1=e_{t \alpha} \otimes \rho_{\alpha} \otimes e_{h \alpha}$ is the $A$-bimodule homomorphism $f_{\alpha}$ determined by

$$
f_{\alpha}(1 \otimes \beta \otimes 1)= \begin{cases}e_{h \alpha} \otimes e_{t \alpha}, & \beta=\alpha, \\ 0, & \text { otherwise. }\end{cases}
$$

We then calculate for each $\beta \in Q_{1}^{\mathrm{m}}$ that

$$
\begin{aligned}
\mu_{2}^{*}\left(f_{\alpha}\right)\left(1 \otimes \rho_{\beta} \otimes 1\right) & =f_{\alpha} \mu_{2}\left(1 \otimes \rho_{\beta} \otimes 1\right) \\
& =f_{\alpha}\left(\sum_{\gamma \in Q_{1}} \Delta_{\gamma}\left(\partial_{\beta} W\right)\right) \\
& =\mathbf{x}_{\beta} \otimes \mathbf{y}_{\beta},
\end{aligned}
$$

where

$$
\Delta_{\alpha}\left(\partial_{\beta} W\right)=\mathbf{x}_{\beta} \otimes \alpha \otimes \mathbf{y}_{\beta} .
$$

We must then have

$$
\Delta_{\beta}\left(\partial_{\alpha} W\right)=\mathbf{y}_{\beta} \otimes \beta \otimes \mathbf{x}_{\beta},
$$

and so the isomorphism $\left(A \otimes \mathbb{K} Q_{2}^{\mathrm{m}} \otimes A, A^{\varepsilon}\right) \stackrel{\sim}{\rightarrow} A \otimes \mathbb{K} Q_{1}^{\mathrm{m}} \otimes A$ takes $\mu_{2}^{*}\left(f_{\alpha}\right)$ to

$$
\sum_{\beta \in Q_{1}^{\mathrm{m}}} \mathbf{y}_{\beta} \otimes \beta \otimes \mathbf{x}_{\beta}=\sum_{\beta \in Q_{1}^{\mathrm{m}}} \Delta_{\beta}\left(\partial_{\alpha} W\right)=\mu_{2}^{\vee}\left(1 \otimes \rho_{\alpha} \otimes 1\right),
$$

as required. 
Now consider the commutative diagram

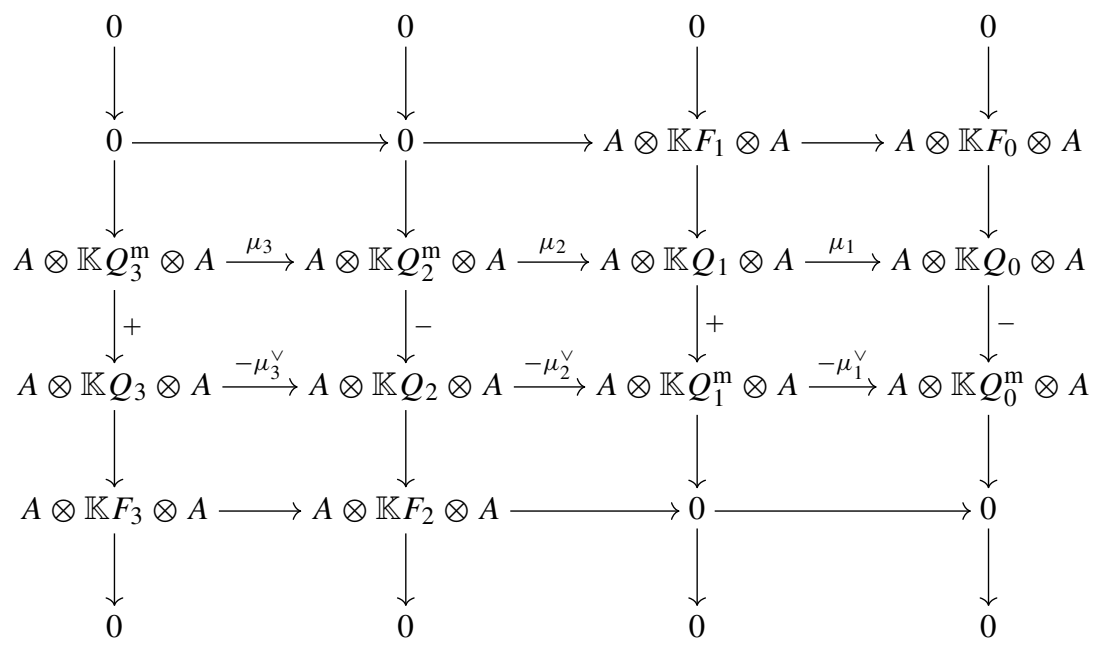

in which the columns are split exact, the second row is $\mathbf{P}(A)$, the third row is isomorphic to $\Omega_{A}[3]$ by the preceding calculations, and the signs on the vertical arrows indicate whether the corresponding map is the inclusion or its negative.

The diagram (5.1) provides us with a map of complexes $A \cong \mathbf{P}(A) \rightarrow \Omega_{A}[3]$ in $\mathcal{D}^{b} A^{\varepsilon}$, and shows that the cone of this map has the form

$$
C=A \otimes \mathbb{K} F_{3} \otimes A \longrightarrow A \otimes\left(\mathbb{K} F_{2} \oplus \mathbb{K} F_{1}\right) \otimes A \longrightarrow A \otimes \mathbb{K} F_{0} \otimes A
$$

Let $M \in \mathcal{D}_{\underline{A}}(A)$ have finite dimensional total cohomology. Since the cohomology of $M$ is concentrated in some interval, $M \in \mathcal{D}^{b} A$. We pick a bounded representative $M^{\bullet}$ of the quasi-isomorphism class of $M$, allowing us to compute the complex $\operatorname{RHom}_{A}(C, M)$ as the total complex of the double complex with terms

$$
\operatorname{Hom}_{A}\left(A \otimes V_{i} \otimes A, M^{j}\right),
$$

where $V_{1}=\mathbb{K} F_{3}, V_{2}=\mathbb{K} F_{2} \oplus \mathbb{K} F_{1}, V_{3}=\mathbb{K} F_{0}$ and $V_{i}=0$ for all other $i$. Since each $S$-bimodule $V_{i}$ has the property that $e V_{i} e=V_{i}$, we have

$$
A \otimes V_{i} \otimes A=A e \otimes V_{i} \otimes e A,
$$

so the terms of the relevant double complex are isomorphic to

$$
\operatorname{Hom}_{S}\left(V_{i} \otimes e A, \operatorname{Hom}_{A}\left(A e, M^{j}\right)\right)=\operatorname{Hom}_{S}\left(V_{i} \otimes e A, e M^{j}\right) .
$$

Since $M \in \mathcal{D}_{A}(A)$, the complex $e M^{\bullet}$ is acyclic. Moreover, since $S$ is semi-simple, $\operatorname{Hom}_{S}\left(V_{i} \otimes e A,-\right)$ preserves acyclicity, so the vertical cohomology of the double complex vanishes. It follows that $\mathbf{R H o m}_{A}(C, M)=0$. A similar argument shows that $\mathbf{R H o m}_{A^{\text {op }}}(C, N)=0$ for all $N \in \mathcal{D}_{\underline{A}^{\text {op }}}\left(A^{\text {op }}\right)$, so we conclude that $A$ is bimodule internally 3-Calabi-Yau with respect to $e$.

Example 5.7 Since the frozen Jacobian algebra $A$ in Examples 2.10 and 5.3 is finite dimensional (of small dimension!) it is possible to check that $\mathbf{P}(A) \stackrel{\sim}{\rightarrow} A$ directly by choosing a basis of $A$, whence this algebra is bimodule internally 3-Calabi-Yau with respect to this 
frozen idempotent. The algebra $A^{\prime}$ in Example 2.10 may also be presented as a frozen Jacobian algebra, and again the fact that $A^{\prime}$ is finite dimensional allows us to check directly that $\mathbf{P}\left(A^{\prime}\right) \stackrel{\sim}{\rightarrow} A^{\prime}$, giving the bimodule internally 3-Calabi-Yau property.

However, we also have many more examples, some of which are infinite dimensional. For example, let

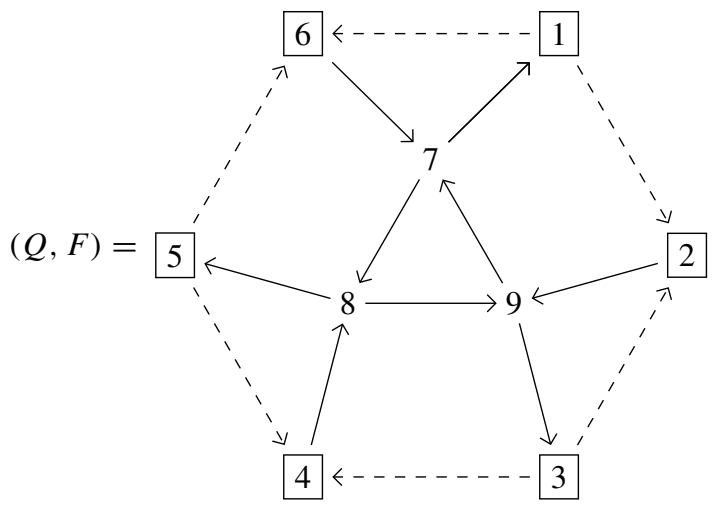

where the frozen subquiver is indicated by boxed vertices and dashed arrows as before, and let $W$ be the potential given by the sum of the 3-cycles minus the sum of the 4-cycles. Writing $\hat{A}=\mathcal{J}(Q, F, W)$, we have $\mathbf{P}(\hat{A}) \stackrel{\sim}{\rightarrow} \hat{A}$. We omit the calculation here, but observe that there is a grading of $\hat{A}$ in which all arrows have positive degree, meaning that by [7, Prop. 7.5] it is sufficient to check that $\mu_{0} \otimes_{\hat{A}} S_{i}: \mathbf{P}(\hat{A}) \otimes_{\hat{A}} S_{i} \rightarrow S_{i}$ is a quasi-isomorphism for each simple $\hat{A}$-module $S_{i}$, which is more straightforward. One can also check that $\hat{A}$ is the endomorphism algebra of a cluster-tilting object in Jensen-King-Su's categorification of the cluster structure on the Grassmannian $G_{2}^{6}$, described in Example 3.12.

The existence of a quasi-isomorphism $\mathbf{P}(A) \stackrel{\sim}{\rightarrow} A$ allows us to deduce many homological properties of $A$ and of the boundary algebra $B$. For example, any $A$-module $M$ has a (usually non-minimal) projective resolution $\mathbf{P}(A) \otimes_{A} M$. Using this, we see immediately that if $M$ is any $A$-module such that $M=e M$, such as a simple module at a frozen vertex, then $A \otimes \mathbb{K} Q_{3}^{\mathrm{m}} \otimes M=0$, and p. $\operatorname{dim}_{A} M \leq 2$.

It follows from Theorem 5.6 that if $A$ is a frozen Jacobian algebra with the property that $\mu_{0}: \mathbf{P}(A) \rightarrow A$ is a quasi-isomorphism, then both $A$ and $A^{\text {op }}$ are internally 3-Calabi-Yau with respect to the frozen idempotent $e$. If additionally $A$ is Noetherian and $A /\langle e\rangle$ is finite dimensional, then we may apply Theorem 4.1. We may also make some observations about the homological algebra of the boundary algebra $B$ in this situation.

Proposition 5.8 (cf. [2, Rem. 2.8]) Let A be a Noetherian frozen Jacobian algebra with frozen idempotent e such that $\mu_{0}: \mathbf{P}(A) \rightarrow A$ is a quasi-isomorphism and $A /\langle e\rangle$ is finite dimensional, and let $B=e$ Ae. Let $\Omega_{B}=\operatorname{RHom}_{B^{\varepsilon}}\left(B, B^{\varepsilon}\right)$. Then $\Omega_{B} \cong e \Omega_{A}$ e in $\mathcal{D}^{b} B^{\varepsilon}$.

Proof Write $P_{i}=A \otimes \mathbb{K} Q_{i} \otimes A$ for $i=0,1$ and $P_{i}=A \otimes \mathbb{K} Q_{i}^{\mathrm{m}} \otimes A$ for $i=2,3$. By Theorem 5.6, $A$ and $A^{\text {op }}$ are internally 3-Calabi-Yau with respect to $e$, so we have $\operatorname{Ext}_{B}^{i}(e A, B)=0=\operatorname{Ext}_{B}^{i}$ op $(A e, B)$ for all $i>0$ by Proposition 4.5. Thus we may calculate

$$
\begin{aligned}
\mathbf{R H o m}_{B^{\varepsilon}}\left(e A \otimes_{\mathbb{K}} A e, B^{\varepsilon}\right) & =\mathbf{R H o m}_{B}(e A, B) \otimes_{\mathbb{K}} \mathbf{R} \operatorname{Hom}_{B^{\text {op }}}(A e, B) \\
& =\operatorname{Hom}_{B}(e A, B) \bigotimes_{\mathbb{K}} \operatorname{Hom}_{B^{\text {op }}}(A e, B)
\end{aligned}
$$




$$
=\operatorname{Hom}_{B^{\varepsilon}}\left(e A \otimes_{\mathbb{K}} A e, B^{\varepsilon}\right) .
$$

It follows that the terms $e P_{i} e$ of the sequence $e \mathbf{P}(A) e \cong B$ satisfy $\operatorname{Ext}_{B^{\varepsilon}}^{i}\left(e P_{i} e, B^{\varepsilon}\right)=0$ for $i>0$, and so

$$
\mathbf{R H o m}_{B^{\varepsilon}}\left(B, B^{\varepsilon}\right) \cong \operatorname{Hom}_{B^{\varepsilon}}\left(e \mathbf{P}(A) e, B^{\varepsilon}\right) .
$$

By Theorem 4.1(iii), the functor $e A \otimes_{A}-\otimes_{A} A e: \operatorname{proj} A^{\varepsilon} \rightarrow \bmod B^{\varepsilon}$ is fully faithful, and so

$$
\operatorname{Hom}_{B^{\varepsilon}}\left(e P_{i} e, B^{\varepsilon}\right) \cong \operatorname{Hom}_{A^{\varepsilon}}\left(P_{i}, A e \otimes_{\mathbb{K}} e A\right)=e \operatorname{Hom}_{A^{\varepsilon}}\left(P_{i}, A^{\varepsilon}\right) e .
$$

It follows that

$$
\begin{aligned}
\Omega_{B} & =\mathbf{R H o m}_{B^{\varepsilon}}\left(B, B^{\varepsilon}\right) \cong \operatorname{Hom}_{B^{\varepsilon}}\left(e \mathbf{P}(A) e, B^{\varepsilon}\right) \\
& =e \operatorname{Hom}_{A^{\varepsilon}}\left(\mathbf{P}(A), B^{\varepsilon}\right) e \cong e \mathbf{R H o m}_{A^{\varepsilon}}\left(A, A^{\varepsilon}\right) e=e \Omega_{A} e .
\end{aligned}
$$

Proposition 5.9 With the notation and assumptions of Proposition 5.8, for any $X \in \mathcal{D}^{b} B$ we have

$$
\Omega_{B} \stackrel{\mathbf{L}}{\otimes}_{B} X \cong X[-3]
$$

in the quotient category $\mathcal{D}^{b} B / \operatorname{per} B \simeq \underline{\mathrm{GP}}(B)$.

Proof The proof of Theorem 5.6 constructs a map $A \rightarrow \Omega_{A}$ [3] with mapping cone

$$
C=A \otimes \mathbb{K} F_{3} \otimes A \longrightarrow A \otimes\left(\mathbb{K} F_{2} \oplus \mathbb{K} F_{1}\right) \otimes A \longrightarrow A \otimes \mathbb{K} F_{0} \otimes A .
$$

Since each $S$-bimodule $\mathbb{K} F_{i}$ has the property that $e\left(\mathbb{K} F_{i}\right) e=\mathbb{K} F_{i}$, we can instead write $C$ as

$$
A e \otimes \mathbb{K} F_{3} \otimes e A \longrightarrow A e \otimes\left(\mathbb{K} F_{2} \oplus \mathbb{K} F_{1}\right) \otimes e A \longrightarrow A e \otimes \mathbb{K} F_{0} \otimes e A
$$

Now applying the functor $e A \otimes_{A}-\otimes_{A}$ Ae to the triangle $A \rightarrow \Omega_{A}[3] \rightarrow C \rightarrow A[1]$ in per $A^{\varepsilon}$ yields the triangle

$$
B \longrightarrow e \Omega_{A} e[3] \longrightarrow e C e \longrightarrow B[1]
$$

in $\mathcal{D}^{b} B^{\varepsilon}$. We have

$$
e C e=B \otimes \mathbb{K} F_{3} \otimes B \longrightarrow B \otimes\left(\mathbb{K} F_{2} \oplus \mathbb{K} F_{1}\right) \otimes B \longrightarrow B \otimes \mathbb{K} F_{0} \otimes B \in \text { per } B^{\varepsilon},
$$

and $e \Omega_{A} e \cong \Omega_{B}$ by Proposition 5.8. So applying $-\stackrel{\mathbf{L}}{\otimes}_{B} X$ to the above triangle yields the triangle

$$
X \longrightarrow \Omega_{B} \stackrel{\mathbf{L}}{\otimes} X[3] \longrightarrow e C e \stackrel{\mathbf{L}}{\otimes} B \longrightarrow X[1]
$$

in $\mathcal{D}^{b} B$. Since $e C e \in$ per $B^{\varepsilon}$, we have $e C e \stackrel{\mathbf{L}}{\otimes_{B}} X \in \operatorname{per} B$, and so $\Omega_{B} \stackrel{\mathbf{L}}{\otimes}_{B} X \cong X[-3]$ in the quotient $\mathcal{D}^{b} B /$ per $B$, by the above triangle. The algebra $B$ is Iwanaga-Gorenstein by Theorem 4.1(a), so $\mathcal{D}^{b} B /$ per $B \simeq \underline{\mathrm{GP}}(B)$ by [11, Thm. 4.4.1]. 
Acknowledgements Open access funding provided by the Max Planck Society. This work is based on part of my Ph.D. thesis [38, §5], which I worked on at the University of Bath under the supervision of Alastair King, and with the support of an Engineering and Physical Sciences Research Council studentship. I am very grateful to Alastair for his support and encouragement. I also thank Charlie Beil, Martin Kalck, Joe Karmazyn and Dong Yang for useful conversations. This paper was revised during a stay at Universität Bielefeld funded by Sonderforschungsbereich 701; I am grateful to Henning Krause and the rest of the representation theory group in Bielefeld for their hospitality. After helpful comments from the referees, it was further revised during a stay at the Max-Planck-Institut für Mathematik in Bonn, funded by the Max-Planck-Gesellschaft.

Open Access This article is distributed under the terms of the Creative Commons Attribution 4.0 International License (http://creativecommons.org/licenses/by/4.0/), which permits unrestricted use, distribution, and reproduction in any medium, provided you give appropriate credit to the original author(s) and the source, provide a link to the Creative Commons license, and indicate if changes were made.

\section{References}

1. Amiot, C.: Cluster categories for algebras of global dimension 2 and quivers with potential. Ann. Inst. Fourier (Grenoble) 59(6), 2525-2590 (2009). arXiv:0805.1035 [math.RT]

2. Amiot, C., Iyama, O., Reiten, I.: Stable categories of Cohen-Macaulay modules and cluster categories. Am. J. Math. 137(3), 813-857 (2015). arXiv:1104.3658 [math.RT]

3. Amiot, C., Reiten, I., Todorov, G.: The ubiquity of generalized cluster categories. Adv. Math. 226(4), 3813-3849 (2011). arXiv:0911.4819 [math.RT]

4. Auslander, M.: Rational singularities and almost split sequences. Trans. Am. Math. Soc. 293(2), 511-531 (1986)

5. Baur, K., King, A.D., Marsh, R.J.: Dimer models and cluster categories of Grassmannians. Proc. Lond. Math. Soc. (3) 113(2), 213-260 (2016). arXiv:1309.6524 [math.RT]

6. Bocklandt, R.: Graded Calabi Yau algebras of dimension 3. J. Pure Appl. Algebra 212(1), 14-32 (2008). arXiv:math/0603558 [math.RA]

7. Broomhead, N.: Dimer models and Calabi-Yau algebras. Memoirs of the American Mathematical Society, vol. 215, no. 1011. American Mathematical Society, Providence (2012). arXiv:0901.4662 [math.AG]

8. Buan, A.B., Iyama, O., Reiten, I., Scott, J.: Cluster structures for 2-Calabi-Yau categories and unipotent groups. Compos. Math. 145(4), 1035-1079 (2009). arXiv:math/0701557 [math.RT]

9. Buan, A.B., Iyama, O., Reiten, I., Smith, D.: Mutation of cluster-tilting objects and potentials. Am. J. Math. 133(4), 835-887 (2011). arXiv:0804.3813 [math.RT]

10. Buan, A.B., Marsh, R.J., Reineke, M., Reiten, I., Todorov, G.: Tilting theory and cluster combinatorics. Adv. Math. 204(2), 572-618 (2006). arXiv:math/0402054 [math.RT]

11. Buchweitz, R.-O.: Maximal Cohen-Macaulay modules and Tate-cohomology over Gorenstein rings (1987). Unpublished manuscript. http://hdl.handle.net/1807/16682

12. Butler, M.C.R., King, A.: Minimal resolutions of algebras. J. Algebra 212(1), 323-362 (1999)

13. Caldero, P., Chapoton, F.: Cluster algebras as Hall algebras of quiver representations. Comment. Math. Helv. 81(3), 595-616 (2006). arXiv:math/0410187 [math.RT]

14. Demonet, L., Iyama, O.: Lifting preprojective algebras to orders and categorifying partial flag varieties. Algebra Number Theory 10(7), 1527-1579 (2016). arXiv:1503.02362 [math.RT]

15. Demonet, L., Luo, X.: Ice quivers with potential associated with triangulations and Cohen-Macaulay modules over orders. Trans. Am. Math. Soc. 368(6), 4257-4293 (2016). arXiv:1307.0676 [math.RT]

16. Dwyer, W.G.: Noncommutative localization in homotopy theory. Non-commutative localization in algebra and topology, pp. 24-39 (2006)

17. Franco, S.: Bipartite field theories: from D-brane probes to scattering amplitudes. J. High Energy Phys. 11 (2012). arXiv:1207.0807 [hep-th]

18. Geiß, C., Leclerc, B., Schröer, J.: Partial flag varieties and preprojective algebras. Ann. Inst. Fourier (Grenoble) 58(3), 825-876 (2008). arXiv:math/0609138 [math.RT]

19. Geiß, C., Leclerc, B., Schröer, J.: Kac-Moody groups and cluster algebras. Adv. Math. 228(1), 329-433 (2011). arXiv:1001.3545 [math.RT]

20. Ginzburg, V.: Calabi-Yau algebras (2007). arXiv:0612139v3 [math.AG]

21. Goncharov, A.B.: Ideal webs, moduli spaces of local systems, and 3d Calabi-Yau categories (2016). arXiv:1607.05228v1 [math.AG]

22. Guo, L.: Cluster tilting objects in generalized higher cluster categories. J. Pure Appl. Algebra 215(9), 2055-2071 (2011). arXiv:1005.3564 [math.RT] 
23. Happel, D.: Triangulated Categories in the Representation Theory of Finite-Dimensional algebras, London Mathematical Society Lecture Note Series, vol. 119. Cambridge University Press, Cambridge (1988)

24. Herzog, J.: Ringe mit nur endlich vielen Isomorphieklassen von maximalen, unzerlegbaren CohenMacaulay-Moduln. Math. Ann. 233(1), 21-34 (1978)

25. Iyama, O.: Auslander correspondence. Adv. Math. 210(1), 51-82 (2007). arXiv:math/0411631 [math.RT]

26. Iyama, O.: Higher-dimensional Auslander-Reiten theory on maximal orthogonal subcategories. Adv. Math. 210(1), 22-50 (2007). arXiv:math/0407052 [math.RT]

27. Iyama, O., Yang, D.: Silting reduction and Calabi-Yau reduction of triangulated categories (2015). arXiv: $1408.2678 \mathrm{v} 3$ [math.RT]

28. Jensen, B.T., King, A.D., Su, X.: A categorification of Grassmannian cluster algebras. Proc. Lond. Math. Soc. (3) 113(2), 185-212 (2016). arXiv:1205.1008v4 [math.AG]

29. Kalck, M., Iyama, O., Wemyss, M., Yang, D.: Frobenius categories, Gorenstein algebras and rational surface singularities. Compos. Math. 151(3), 502-534 (2015). arXiv:1209.4215 [math.RT]

30. Kalck, M., Yang, D.: Relative singularity categories I: Auslander resolutions. Adv. Math. 301, 973-1021 (2016). arXiv:1205.1008 [math.AG]

31. Keller, B.: Calabi-Yau Triangulated Categories. In: Trends in representation theory of algebras and related topics, pp. 467-489 (2008)

32. Keller, B.: Cluster algebras, quiver representations and triangulated categories. In: Triangulated categories. pp. 76-160 (2010). arXiv:0807.1960 [math.RT]

33. Keller, B., Reiten, I.: Cluster-tilted algebras are Gorenstein and stably Calabi-Yau. Adv. Math. 211(1), 123-151 (2007). arXiv:math/0512471 [math.RT]

34. Leuschke, G.J., Wiegand, R.: Cohen-Macaulay Representations, Mathematical Surveys and Monographs, vol. 181. American Mathematical Society, Providence (2012)

35. Nájera Chávez, A.: A 2-Calabi-Yau realization of finite-type cluster algebras with universal coefficients (2016). arXiv:1512.07939v2 [math.RT]

36. Palu, Y.: Cluster characters for 2-Calabi-Yau triangulated categories. Ann. Inst. Fourier (Grenoble) 58(6), 2221-2248 (2008). arXiv:math/0703540 [math.RT]

37. Postnikov, A.: Total positivity, Grassmannians, and networks (2006). arXiv:math/0609764v1 [math.CO]

38. Pressland, M.: Frobenius Categorification of Cluster Algebras. Ph.D. thesis (2015). http://opus.bath.ac. uk/49079/

39. Reiten, I., Van den Bergh, M.: Two-dimensional tame and maximal orders of finite representation type. Memoirs of the American Mathematical Society, vol. 408. American Mathematical Society, Providence (1989)

40. Weibel, C.A.: An Introduction to Homological Algebra, Cambridge Studies in Advanced Mathematics, vol. 38. Cambridge University Press, Cambridge (1994) 